\title{
WiFi and LAA Coexistence: Improvements for WiFi's Performance
}

\author{
by
}

\begin{abstract}
Aliasghar Keyhanian, B.Sc.
A thesis submitted to the

Faculty of Graduate and Postdoctoral Affairs

in partial fulfillment of the requirements for the degree of
\end{abstract}

Master of Applied Science in Electrical Engineering

Ottawa-Carleton Institute for Electrical and Computer Engineering

Department of Systems and Computer Engineering

Carleton University

Ottawa, Ontario

August, 2017

(C) Copyright

Aliasghar Keyhanian, 2017 
The undersigned hereby recommends to the

Faculty of Graduate and Postdoctoral Affairs acceptance of the thesis

\title{
WiFi and LAA Coexistence: Improvements for WiFi's Performance
}

\author{
submitted by Aliasghar Keyhanian, B.Sc. \\ in partial fulfillment of the requirements for the degree of \\ Master of Applied Science in Electrical Engineering \\ Professor "A. Nayak" \\ Professor I. Lambadaris, Thesis Supervisor \\ Professor "I. D. Marsland" \\ Professor Roshdy Hafez, Committee Chair, \\ Department of Systems and Computer Engineering \\ Ottawa-Carleton Institute for Electrical and Computer Engineering \\ Department of Systems and Computer Engineering \\ Carleton University
}

August, 2017 


\section{Abstract}

As the demand for mobile data traffic increased significantly in the past few years, the Mobile Network Operators (MNOs) are considering different solutions to increase the network capacity. Since the licensed spectrum is scarce and expensive, deploying LTE in the unlicensed band can be a promising method to accommodate the market need for mobile data. However, LTE has to coexist with other heterogeneous network, especially WiFi, preexisting in the unlicensed medium. This coexistence can introduce a significant performance degradation for currently deployed WiFi networks. In this study, emphasis is given in analyzing the performance of WiFi and LTE coexistence scenario both analytically and through simulation. To this end, a number of solutions are proposed to increase the performance of WiFi.

Firstly, a numerical analysis is performed to mathematically formulate the problem of coexistence between WiFi and LTE. Results show that LTE can outperform $\mathrm{WiFi}$ in terms of gaining access to the medium. Therefore, several modifications in the WiFi network have been studied (e.g. maximum packet length) to allow the WiFi network to achieve a fair share of the bandwidth while coexisting with LTE. The study is complemented with a heuristic approach that allows to efficiently calculate the payload length of WiFi packets. Specifically, the packet size is being determined on the fly according to the number of existing WiFi and LTE communicating devices. Finally, an alternative approach to compensate for the WiFi degradation in a coexistence scenario is proposed based on link adaptation. In particular, a number

of enhancements in WiFi link adaptation is proposed to increase its adaptability in case of coexistence with LAA-LTE. 
I want to dedicate this thesis to my parents Maryam and Ali who have always encouraged me for new adventures like this one. I wish that this accomplishment make your dreams come true when you tried your best to give me the best education possible. 


\section{Acknowledgments}

First of all, I am thankful to the merciful God for helping me to begin, continue and finish my work.

I would like to acknowledge the help and support of Dr. I. Lambadaris, my thesis supervisor, for guiding me in my research area and helping me complete my program requirements. I would also wish to express my sincere thanks to Dr. A. Leivadeas for providing me extra academic support to achieve my research goals.

Finally, I express my sincere thanks to my parents for all of their encouragements towards the completion of this study. 


\section{Preface}

This thesis is the final work of my Master of Science program at the department of System and Computer Engineering in Carleton University from the Fall 2015 until the Summer 2017. It displays my results for the Coexistence of WiFi and LTE networks in the unlicensed spectrum. This emerging coexistence is very essential to expand the network capacity. Therefore it is my passion to try to facilitate this coexistence in my study.

In fact, I could not achieve my current success without the help of a few number of people. First, I am very grateful for the academic and financial support of my supervisor, Dr. I. Lambadaris, toward the completion of my program. Second, I would like to express my deep appreciation for the academic help and guidance of Dr. A. Leivadeas. 


\section{Table of Contents}

Abstract $\quad$ iii

Acknowledgments $\quad$ v

Preface $\quad$ vi

Table of Contents vii

List of Tables $\quad$ ix

List of Figures $\quad \mathrm{x}$

1 Introduction $\quad 1$

1.1 LTE-U and LAA Operations . . . . . . . . . . . . . . . 3

1.2 Link Adaptation . . . . . . . . . . . . . . . . . . 4

1.3 Thesis Contribution . . . . . . . . . . . . . . . 6

1.4 Thesis Organization . . . . . . . . . . . . . . . 7

2 Related Work $\quad 8$

2.1 Coexistence of Heterogeneous Networks . . . . . . . . . . . . . . 8

2.2 WiFi Link adaptation . . . . . . . . . . . . . . . . 11

2.2.1 SNR based Link Adaptation . . . . . . . . . . . . . . . . . . . 11

2.2.2 Frame loss based Link Adaptation . . . . . . . . . . . . . . . . 12

2.3 Link Adaptation in LTE . . . . . . . . . . . . . . . . . . . . . . 14

3 Theoretical Evaluations 17

3.1 WiFi Performance without Interference . . . . . . . . . . . . 17

3.2 Coexistence Performance of WiFi and LAA . . . . . . . . . . 20

3.2.1 Analytical Evaluation for LBT . . . . . . . . . . . 21 
3.2.2 Analytical Evaluation of Coexistence . . . . . . . . . . . . . 22

3.3 Numerical Analysis of WiFi and LAA Coexistence . . . . . . . . . . . 24

3.3.1 Performance Analysis for Fair Coexistence of WiFi and LTE-LAA 26

3.4 Allocation of Users between the two RANs . . . . . . . . . . . . . 32

4 Link Adaptation Considerations 35

4.1 Overview of Matlab Simulator . . . . . . . . . . . . . . . 35

4.2 Original Minstrel Algorithm . . . . . . . . . . . . . . . . . 38

4.2.1 Retry Chain . . . . . . . . . . . . . . . . . . 38

4.2 .2 Rate Selection . . . . . . . . . . . . . . . 38

4.2.3 Statistics Calculation . . . . . . . . . . . . . . . 38

4.3 Enhanced Minstrel Implementation . . . . . . . . . . . . . . . . 39

4.4 Mutual Information Implementation . . . . . . . . . . . . . . . . . 41

4.5 Results and Discussions . . . . . . . . . . . . . . . 43

4.5.1 Performance Verification of Link Adaptation in WiFi (1 AP 1 $\mathrm{STA}$. . . . . . . . . . . . . . . . . . 44

4.5.2 Performance Verification of Link Adaptation in WiFi (1 AP 5 stations) . . . . . . . . . . . . . . . 45

4.5.3 Performance Evaluation of WiFi and LAA coexistence (1 $\mathrm{AP} / \mathrm{BS} 1$ station/UE) . . . . . . . . . . . . 46

4.5.4 Performance Evaluation of $\mathrm{WiFi}$ and LAA coexistence (1 $\mathrm{AP} / \mathrm{BS} 5$ stations/UEs) . . . . . . . . . . . . 47

4.5.5 Equalizing performance in WiFi and LAA coexistence . . . . . 49

5 Conclusion and Future Works 54

$\begin{array}{ll}\text { List of References } & 56\end{array}$ 


\section{List of Tables}

1.1 MCS Index, Modulation Scheme and coding scheme . . . . . . . . 6

2.1 The 4-BIT CQI Table in LTE . . . . . . . . . . . . . . . . 16

3.1 Transmission and collision probability of WiFi and LAA for different number of stations in each network . . . . . . . . . . . . . . . . . 27

3.2 Simulation Parameters . . . . . . . . . . . . . . . . . 33

4.1 Minstrel Retry Chain ( $\mathrm{T}$ : highest throughput, t: second highest throughput, P: highest probability, B: Base Rate, S: sample rate) . 39

4.2 Starting Rate to SNR association . . . . . . . . . . . . . . 40

4.3 Enhanced Minstrel Retry Chain (T: highest throughput, t: second highest throughput, P: highest probability, P-1: Second highest probability, S: sample rate) . . . . . . . . . . . . . . . . 41 


\section{List of Figures}

1.1 Constellation Diagrams for Different Modulation Schemes . . . . . . . 5

2.1 Link Adaptation in LTE Operation . . . . . . . . . . . . . . . . 15

3.1 Markov chain model for the CSMA/CA [1] . . . . . . . . . . . 18

3.2 Time required for a successful transmission . . . . . . . . . . . . . . 19

3.3 Time required for a collision . . . . . . . . . . . . . . . 20

3.4 Markov chain model for the Cat4 LBT [2] . . . . . . . . . . . 21

3.5 System model for the coexistence of WiFi and LTE. Solid connectors show the current attachments and dashed connectors display the potential connections. . . . . . . . . . . . . . . 23

3.6 Comparison of transmission probability of WiFi and LAA . . . . . 25

3.7 Comparison of collision probability of WiFi and LAA . . . . . . . . . 26

3.8 Packet length ratio of WiFi over LAA for having equal throughput. The number of users for both networks are the same. . . . . . . . . . 28

3.9 Average throughput difference of WiFi and LAA for different numbers of $\alpha$ within $(-2,2)$ and $c$ within $(0,2)$. Fairness criterion: WiFi throughput $=$ LAA throughput . . . . . . . . . . . . .

3.10 LAA/WiFi average throughput ratio for changing packet size based on heuristic approach versus the fixed packet size (LAA users are varying from 3 to 9 and the ratio is the average of all cases). Fairness criterion: WiFi throughput $=$ LAA throughput . . . . . . . . . . . .

3.11 Average throughput difference of WiFi and LAA for different numbers of $\alpha$ within $(-2,2)$ and $c$ within $(0,2)$. Fairness criterion: WiFi throughput per number of station $=$ LAA throughput per number of station . . . . . . . . . . . . . . . . . 
3.12 LAA/WiFi average throughput ratio for changing packet size based on heuristic approach versus the fixed packet size (LAA users are varying from 3 to 9 and the ratio is the average of all cases). Fairness criterion: WiFi throughput per number of station $=$ LAA throughput per number

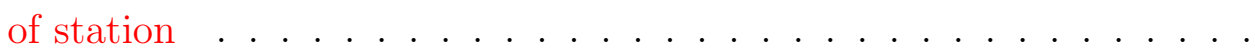

3.13 Comparison of three approaches: i)Random allocation of users, ii)Optimal allocation of users upon arrivals, iii) Optimal allocation of users upon both arrivals and departures . . . . . . . . . . . . 34

4.1 SNR to Mutual Information Mapping . . . . . . . . . . . . . 42

4.2 Minstrel performance in terms of a) Throughput and b) PER with increasing distance . . . . . . . . . . . . .

4.3 Minstrel performance in terms of a) Throughput and b) PER with increasing distance . . . . . . . . . . . . .

4.4 WiFi and LAA coexistence performance in terms of a) Throughput and b) PER with increasing distance . . . . . . . . . .

4.5 WiFi and LAA coexistence performance in terms of a) Throughput and b) PER with increasing distance . . . . . . . . . . . 48

4.6 WiFi and LAA coexistence performance in terms of a) Throughput and b) PER with increasing offset distance between AP and BS ...

4.7 WiFi and LAA coexistence performance in terms of a) Throughput and b) PER with LAA sending 2 subframes. . . . . . . . . .

4.8 WiFi and LAA coexistence performance in terms of a) Throughput and b) PER with LAA sending 1 subframes. . . . . . . . . . .

4.9 WiFi and LAA coexistence performance in terms of a) Throughput and b) PER with no link adaptation activated and with equal transmission times . . . . . . . . . . . . . . . . . .

4.10 WiFi and LAA coexistence performance in terms of a) Throughput and b) PER with link adaptation activated and with equal transmission times 


\section{List of Acronyms}

\begin{tabular}{ll} 
AARF & Adaptive Auto Rate Fallback \\
ABS & Almost-Blank Subframe \\
ACK & Acknowledgement \\
AMC & Adaptive Modulation and Coding \\
AP & Access Point \\
ARF & Auto Rate Fallback \\
A-RTS & Adaptive Ready to send \\
AWGN & Additive White Guassian Noise \\
BARA & Beacon Auto Rate Adaptation \\
BEB & Binary Exponential Backoff \\
BLER & Block Error Rate \\
BPSK & Binary Phase Shift Keying \\
BS & Base Station \\
CARA & Collision Aware Rate Adaptation \\
CCA & Clear Channel Assessment \\
CQI & Channel Quality Indicator \\
CSAT & Carrier Sense Adaptive Transmission \\
CS & Carrier Sense \\
CSI & Channel State Information \\
CSMA/CA & Carrier Sense Multiple Access with Collision Avoidance \\
CTS & Clear to Send \\
CW & Contention Window \\
\hline
\end{tabular}




\begin{tabular}{|c|c|}
\hline DCF & Distributed Coordination Function \\
\hline DIFS & Distributed Coordination Function Interframe Space \\
\hline DL & Downlink \\
\hline ECCA & Extended CCA \\
\hline EDCA & Enhanced Distributed Channel Access \\
\hline eICIC & enhanced Inter-Cell Interference Coordination \\
\hline eNB & Evolved Node B \\
\hline ETSI & European Telecommunications Standards Institute \\
\hline EWMA & Exponential Weighting Moving Average \\
\hline FDD & Frequency Division Duplexing \\
\hline FEC & Forward Error Correction \\
\hline LAA & License Assisted Access \\
\hline LBT & Listen before Talk \\
\hline LTE & Long Term Evolution \\
\hline LTE-U & LTE Unlicensed \\
\hline MAC & Media Access Control \\
\hline MCS & Modulation and Coding scheme \\
\hline MI & Mutual Information \\
\hline MNO & Mobile Network Operator \\
\hline NACK & Negative Acknowledgement \\
\hline OAR & Opportunistic Auto Rate \\
\hline OFDM & Orthogonal Frequency Division Multiplexing \\
\hline OFDMA & Orthogonal Frequency Division Multiple Access \\
\hline PAPR & Peak-to-Average Power Ratio \\
\hline PER & Packet Error Rate \\
\hline QAM & Quadrature Amplitude Modulation \\
\hline QPSK & Quadrature Phase Shift Keying \\
\hline RAN & Radio Access Network \\
\hline
\end{tabular}




\begin{tabular}{ll} 
RB & Resource Block \\
RBAR & Receiver Based Auto Rate \\
RF & Radio Frequency \\
RLAN & Radio Local Area Network \\
RTS & Ready to Send \\
SC-FDMA & Single Carrier Frequency Division Multiple Access \\
SDN & Software Defined Networking \\
SIFS & Short Interframe Space \\
SINR & Signal to Interference and Noise Ratio \\
SNR & Signal to Noise Ratio \\
TBS & Transport Block Size \\
TDD & Time Division Duplexing \\
TDM & Time Division Multiplexing \\
UE & User Equipment \\
UL & Uplink \\
WiFi MU & WiFi Medium Utilization \\
WLAN & Wireless Local Area Network \\
\hline
\end{tabular}




\section{Chapter 1}

\section{Introduction}

Currently, the demand for mobile data traffic is growing too fast. Accommodating this growth, enforced the Mobile Network Operators (MNOs) to investigate different solutions to increase the network capacity. Licensed wireless medium is too expensive and the physical layer is reaching its theoretical potential. Therefore, using the unlicensed spectrum is deemed unavoidable to meet the market needs for mobile communication. Previously, this usage was limited to offloading the mobile traffic onto WiFi networks which have ubiquitous deployment and comparably low cost. Recently, besides the data offloading, mobile operators are also looking to operate LTE in the unlicensed spectrum, an approach known as LTE unlicensed (LTE-U) [3] or License-Assisted Access (LAA). However, this deployment can have a huge impact on the operation of $\mathrm{WiFi}$ and introduces several challenges for both networks to gratefully coexist in a shared spectrum. Understanding the coexistence challenges for both WiFi and LTE networks requires a closer look in their operation in the Physical and Mac Layers.

In a LTE network, User Equipment (UE) is synchronous to the base station or eNB. Wireless communication in Downlink (DL) and Uplink (UL) can be performed simultaneously or sequentially whether Frequency Division Duplexing (FDD) mode or Time Division Duplexing (TDD) method is followed [4]. In the physical layer, LTE uses Orthogonal Frequency Division Multiple Access (OFDMA) technology in the downlink direction allowing the transmission to several users at the same time. This technology enables the eNB to determine the resource block allocation to multiple users in the frequency-time space. This technique can increase the throughput significantly since every user in the LTE network is experiencing varying channel conditions over different frequency-time blocks. Since UEs are more vulnerable to power 
consumption due to their dependence on batteries, LTE employs Single Carrier Frequency Division Multiple Access (SC-FDMA) technology in the uplink transmission. SC-FDMA offers a lower Peak-to-Average Power Ratio (PAPR) which enhance mobile devices to have a lower cost for power amplifiers and a better transmit power efficiency.

Unlike the LTE, accessing the medium by WiFi is contention based utilizing the Distributed Coordination Function (DCF) or Enhanced Distributed Channel Access (EDCA) procedure. DCF and EDCA use Carrier Sense Multiple Access with Collision Avoidance (CSMA/CA) protocol accompanied by a random back off time in a busy medium situation [4]. The goal of CSMA/CA is to decrease the collision probability among multiple stations trying to access the medium. If a station wants to transmit a block of data it should sense the medium to specify that whether the medium is busy or idle. Carrier sense (CS) mechanism determines the state of the medium (busy or idle) through physical and virtual Carrier Sense functions. If the medium determined to be idle DCF or EDCA operation may continue. Otherwise the transmitting station should wait until the end of the current transmission. WiFi also transmit beacon frames periodically to broadcast the presence of a Wireless Local Area Network (WLAN). Beacons also follow the CSMA/CA procedure to access the channel. When the number of competing stations increases, the collision probability will get closer to one resulting in a poor performance compared to the centralized LTE operation. Moreover, WiFi physical layer uses Orthogonal Frequency Division Multiplexing (OFDM) technology for both DL and UL directions. OFDM used in WiFi has a different subcarrier granularity and spacing from the LTE physical layer making their waveforms non-orthogonal. This non-orthogonality can further cause interference problems within different carriers in the shared spectrum.

Both networks need to coexist peacefully in the shared medium to achieve the highest performance and lowest interference. Therefore, the LTE operation should be revised in order not to degrade the performance of WiFi network to an unacceptable level. Qualcomm originally developed LTE-U as a revision to LTE operation while Ericsson uses LAA to introduce similar technology. Mobile operators are interested in these two technologies to boost the capacity and coverage of the LTE network by carrier aggregation. Users on the other hand benefit from LTE-U and LAA through the increase in their peak data ratio. Both technologies try to break the LTE cycling in some periods of time allowing WiFi to transmit in the free medium. These two 
technologies are introduced in the following.

\section{$1.1 \quad$ LTE-U and LAA Operations}

LTE-U small cells will search the unlicensed spectrum to find the least busy channel available for offloading their traffic when the licensed spectrum is not sufficient [5]. Also in the case of interference in the operating channel, LTE-U will switch to another clean channel to continue the transmission. LTE-U uses Carrier Sensing Adaptive Transmission (CSAT) algorithm when there is no free channel available for transmission and the load of system is high (high data traffic). This algorithm enables Time Division Multiplexing (TDM) to share a same channel with other Access Points (APs). CSAT also tries to bring fairness in the sharing of the channel with other neighboring WiFi APs and avoid dominating the channel. For transmitting in a TDM manner, CSAT will observe the medium for a comparably long period (from tens of milliseconds up to $200 \mathrm{~ms}$ ) to estimate the WiFi utilization and based on the observation, the algorithm will switch the LTE cycling on and off. More specifically, a time cycle will be defined by CSAT to allow transmission for a certain time periods. In the remaining time of the cycle, LTE will be silent to permit the communication for other neighboring APs. Furthermore LTE transmission time, $T_{o n}$, will be updated according to WiFi Medium Utilization (WiFi MU). If WiFi MU is larger than a certain threshold, $T_{\text {on }}$ will be decremented until reaching the $T_{\text {on min }}$. Otherwise, $T_{\text {on }}$ will be increased until the $T_{o n, \max }$ bound. $T_{o n, \min }$ is defined to ensure that LTE will receive a fair share of the medium and $T_{o n, \max }$ allows CSAT to sense the WiFi activity [6]. The main difference of the CSAT algorithm with CSMA protocol used in WiFi is the longer latency caused by avoiding channels which is currently in the usage of WiFi. CSAT is compatible with the release of 10/11 UE Physical and MAC layer standards [7].

On the other hand, LAA uses Listen Before Talk (LBT) technique to resolve the accessing problem in the coexistence of WiFi and LTE. LBT uses Clear Channel Assessment (CCA) procedure to resolve the contention for accessing the medium. In this procedure the energy level on the channel is estimated before the transmission to identify whether the medium is busy or idle. Starting the transmission requires that the medium is sensed idle for a random back-off phase consisting of N CCA periods. Every CCA period has a $9 \mu$ s duration. Number $\mathrm{N}$ refers to a counter selected randomly from the contention window interval $(0, C W)$. The back-off timer 
decrements after each CCA idle period. In the case that the energy is detected to be greater than a specific threshold while in the back-off procedure, the counter freezes. The counter can resume the decreasing only when the medium is sensed idle for a defer-period. Defer-period is made up of a silent duration similarly with the Distributed Coordination Function Interframe Space (DIFS) period in WiFi. If the transmission is unsuccessful (receiving a Negative Acknowledgement (NACK)) the contention window will be doubled to reach a predefined maximum level. Else, the contention window will reset to the minimum value [8]. After the completion of the random back-off and the subsequent transmission, a new random back-off will be chosen from the contention window for the next transmission.

Some regions like Japan and Europe mandated applying specific LBT operations for LTE transmission in the unlicensed band. For example, The European Telecommunications Standards Institute (ETSI) that places limitations on Radio Local Area Network (RLAN) has specified CCA requirements for different groups of equipment. On the other hand, in the United States, China and Korea there are no mandated CCA requirements for LTE and WiFi coexistence in the unlicensed medium.

One important feature in both WiFi and LTE networks is the link adaptation technique. In the following, this technique is described and indicated how it enables the wireless networks to improve their performance. One of the thesis goal is to enhance this technique for the WiFi network to facilitate the WiFi and LTE coexistence in the shared spectrum.

\section{$1.2 \quad$ Link Adaptation}

Link Adaptation for wireless transmissions can lead to a comparable increase in throughput and higher spectrum efficiency. Link Adaptation algorithms main intention is to adapt to channel variation by changing link parameters such as Modulation and Coding scheme (MCS) and transmission power [9]. Data bits can be modulated with different MCS representing various degrees of robustness and efficiency. Higher MCS has higher efficiency in terms of throughput. Thus, there are more bit patterns and symbols in a same carrier signal. This is done by sharper encoding of the frequency, phase and amplitude. Increasing the efficiency comes with the cost of lower robustness, making the carrier signal more vulnerable to poor channel condition and 


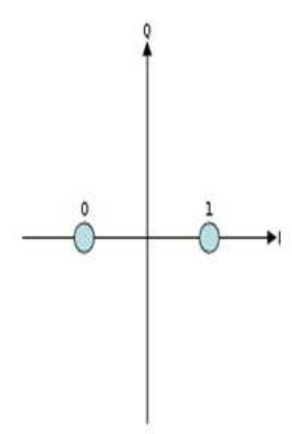

(a) BPSK

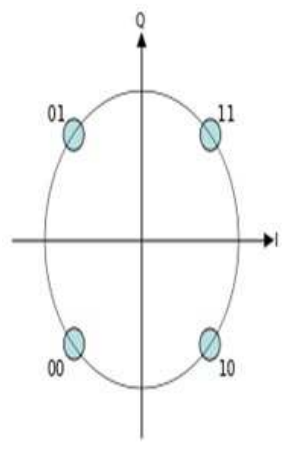

(b) QPSK

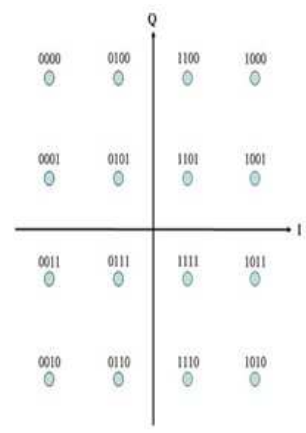

(c) $16 \mathrm{QAM}$

Figure 1.1: Constellation Diagrams for Different Modulation Schemes

signal fading [10]. Therefore, to achieve the best performance the MCS should be chosen dynamically based on the variation of channel condition. Link Adaptation has been studied for a long time because of its role in improving network performance. The freedom degree for most algorithms is the different MCSs available for a given transmission. Usually, when the Signal to Interference and Noise Ratio (SINR) is measured, the highest MCS satisfying the measured value will be chosen.

There are different modulation schemes for wireless transmissions. For example, Binary Phase Shift Keying (BPSK) or Quadrature Phase Shift Keying (QPSK) can be used for noisier channels and 16-QAM when the noise level is lower. In the QPSK modulation, there are two bits per symbol while in the 16-QAM there are four. Therefore, 16-QAM has twice the rate of QPSK with a trade-off of more vulnerability to interference and noise which makes the communication less robust. Wireless devices use different modulations to adapt to the channel conditions. In Figure 1.1, different constellations for three Modulation and Coding Schemes are illustrated by showing possible symbols chosen by a specific Modulation in the complex plane.

On the other hand, different coding schemes will be applied to the modulation formats. In order for the receiver to decode the information correctly some data are needed to be punctured to the original information. This redundancy will help the correct transmission of the original signal. Receiver will check whether the redundancy is compatible to the useful information or not. Also, some errors can be corrected without the need of retransmission using the Forward Error Correction (FEC) method. FEC will employ different coding rate such as $1 / 2,2 / 3,3 / 4$. In the code rate of $2 / 3$, 
two out of three bits is useful information and one bit is added redundancy. In the high channel quality case the coding rate will be close to one reducing the redundant bits to achieve a higher goodput (number of useful bits transmitted to a receiver per unit of time. Useful bits exclude the overheads and added bits for encoding). The different modulation and coding schemes used in WiFi communication is shown in the Table 1.1. As it can be seen from the Table, different coding can be applied to the same modulation scheme.

Table 1.1: MCS Index, Modulation Scheme and coding scheme

\begin{tabular}{c|c|c} 
MCS Index & Modulation Scheme & Coding Scheme \\
\hline \hline 0 & BPSK & $1 / 2$ \\
\hline 1 & QPSK & $1 / 2$ \\
\hline 2 & QPSK & $3 / 4$ \\
\hline 3 & $16-\mathrm{QAM}$ & $1 / 2$ \\
\hline 4 & $16-\mathrm{QAM}$ & $3 / 4$ \\
\hline 5 & $64-\mathrm{QAM}$ & $2 / 3$ \\
\hline 6 & $64-\mathrm{QAM}$ & $3 / 4$ \\
\hline 7 & $64-\mathrm{QAM}$ & $5 / 6$ \\
\hline 8 & $256-\mathrm{QAM}$ & $3 / 4$ \\
\hline 9 & $256-\mathrm{QAM}$ & $5 / 6$
\end{tabular}

\subsection{Thesis Contribution}

In this work, the coexistence of WiFi and LTE in the case that LTE is operating under LAA protocol has been studied. Moreover, by increasing the WiFi's packet payload dynamically and through optimization, a higher level of fairness is tried to be achieved. Furthermore, link adaptation algorithms associated with these technologies are explored emphasizing on enhancing the WiFi's link adaptation performance in the case of coexistence. The main contributions of this thesis are as follows: 
1. Changing the WiFi packet payload optimally and heuristically to achieve fairness in the saturation condition.

2. Allocation of incoming users between WiFi and LTE in order to minimize the performance gap within the two technologies.

3. Proposing enhancements in WiFi link adaptation technique to facilitate the coexistence.

\subsection{Thesis Organization}

In the next chapter the related work in the field of coexistence of Heterogeneous Networks is studied focusing mainly on LTE and WiFi. These works are accompanied by the researches performed in the link adaptation technique for WiFi and LTE technologies. In Chapter 3, the theoretical evaluation of WiFi and LTE are discussed. Moreover, the numerical analysis of the fair coexistence of WiFi and LTE is presented. Chapter 4 studies specifically the Minstrel algorithm with some applied changes. Furthermore, some features of the LTE link adaptation algorithm, called Mutual Information, are explored. Moreover, two sets of experiments are performed and the associated results are discussed. Finally, in Chapter 5 the conclusion and future works are presented. 


\section{Chapter 2}

\section{Related Work}

In this chapter, the background work in the concept of coexistence of heterogeneous networks is reviewed targeting the focus on the coexistence of WiFi and LTE networks. Furthermore, link adaptation technique as a improving network performance tool is explored both in WiFi and LTE.

\subsection{Coexistence of Heterogeneous Networks}

The coexistence of heterogeneous networks operating in a shared spectrum has been well studied in the literature from different aspects. In [11] the coexistence of WiFi with Radio Frequency (RF) interferences from devices like Bluetooth headsets and cordless phones has been investigated. The research shows that 802.11 network is very vulnerable to interferences of other devices activating in its bandwidth. Some extreme cases showed that links in WiFi networks can be interrupted with signals which have only 1/1000 power level of 802.11 signals. In [12] the coexistence of WiFi and ZigBee is under consideration. ZigBee is considered a big source of interference for WiFi communication. One major problem in this coexistence is that WiFi APs are unable to identify the transmission of ZigBee devices since the transmitted packets in ZigBee has a $20 \mathrm{~dB}$ lower power compared to WiFi packets. The unawareness of their coexistence will result in a big probability of collision resulting in a poor performance for both networks. Furthermore, the coexistence between WiFi and WiMAX has been studied in [13] focusing on coexistence interference problems due to simultaneous operation of several radio frequencies.

Coexistence of LTE and WiFi without any modification to both networks has been studied in a paper [14] published by Nokia Research. In this work, LTE and 
WiFi operation in the shared spectrum has been evaluated in an office environment in $900 \mathrm{MHz}$ frequency close to the TV White Space band. Their simulation experiment included offices with single floor and multi floor environments and different densities for WiFi and LTE nodes. Authors found out that the WiFi performance will be degraded significantly in the presence of LTE operating in the same band. On the contrary, LTE performance was not affected in the presence of WiFi achieving almost the same throughput as before. They have presented that this kind of behavior from WiFi network is due to the CSMA protocol running on WiFi nodes. CSMA enforces WiFi to be in the Listen mode around $96 \%$ of the times in most scenarios. In this approach WiFi users will starve and LTE users dominate the channel. Moreover, Babaei et al have proposed probabilistic and numerical analysis of this coexistence [4]. Their study showed that without changing the existing protocols for compatibility, WiFi will end up being in the listening mode with a big probability. In [15] authors evaluated the coexistence of LTE and WLAN. They have concluded that although this coexistence has a negative impact on WLAN network, the severity of the negative influence is manageable by limiting the LTE activity.

Improving the WiFi and LTE coexistence also has been addressed in several studies. The authors in [16] investigate the concept of deploying LTE into an unlicensed band (WiFi spectrum) utilizing the LTE pico-cells. Authors try to reuse the carriers by introducing an underlay of low power nodes in the macro-cell. They considered a type of LBT technique to modify LTE to coexist peacefully with WiFi. Performance analysis displayed the ability of LTE pico-cells to deliver significant amount of throughput even when the LTE pico-cells were sharing their spectrum with WiFi. In [17] a physical layer framework for simultaneous LTE and WiFi transmission and reception has been presented. In the proposed framework the Block Error Rate (BLER) for WiFi is slightly larger when the LTE causes the interference compared to interferences from another WiFi device. Their simulation results also uncovered that by decreasing the LTE bandwidth from $20 \mathrm{MHz}$ to $5 \mathrm{MHz}$, the Signal to Noise (SNR) ratio of WiFi improves by $2.5 \mathrm{~dB}$. Chavez et al introduce the idea of using LTE UL power control to increase the LTE/WiFi coexistence performance [18]. In this study an additional power control mechanism is introduced for UEs to reduce their transmission power. This reduction in transmitted power can significantly reduce the interference within these two networks. Their simulation results shows that utilizing 
this power control can result in a performance as good as when LTE sub-frames are reserved for WiFi only transmissions. Sagari et al proposed a structure for coordination between WiFi and LTE [19]. In this framework, information is exchanged within the two networks to reach a centralized optimization for managing the spectrum. This optimization is based on the architecture of Software Defined Networking (SDN). SDN structure can manage the spectrum dynamically involving several autonomous networks supporting the exchange of information required for heterogeneous networks coordination. This centralized approach can result in an improvement in the overall throughput achieved by the two networks and was validated with the simulation results.

In LTE release 10 an important feature, Almost-Blank Subframe (ABS), was introduced. ABS is a subframe with lower downlink transmission power intended for enhanced Inter-Cell Interference Coordination (eICIC) purposes. This feature can enable WiFi and LTE coexistence in a TDM manner as shown in [20]. The authors are using a refined version of ABS that excludes reference signals resulting in a silent or blank subframe. WiFi can detect channel vacancy during these blank subframes and find a good opportunity to transmit. Their results indicate that this approach can increase the WiFi performance in this coexistence. Furthermore, Zhang et al [21] tried to improve the ABS mechanism by adding an interference avoidance scheme. This scheme uses an interference evaluation technique without the need of information sharing among Base Stations (BSs) and UEs. Number of co-channel transmitters is estimated by the BS with sensing the spectrum. Then the average channel quality will be computed based on the co-channel transmitters and cell density in the area [22]. Simulation results prove that system performance improves by using this method.

Finally, fair coexistence of WiFi and LTE has been investigated in different studies. Cano et al [23] compared the level of fairness of CSAT and LBT techniques when coexisting with WiFi. Their study reveals that if these two techniques are optimally configured, they can provide a same degree of fairness to WiFi. However, they claim that for shorter LTE transmissions, LBT is more efficient. Since the CSAT approach for reserving the channel leads to a higher LTE/WiFi collision probability. Authors in [24] proposed a proportional fair allocation scheme for the LTE/WiFi coexistence. In this research the on-off LTE cycling dynamically changes to ensure the LTE/WiFi proportional fair allocation. Moreover, [25] suggested max-min fairness to 
optimize the LTE-off cycling periods to maximize the minimum weighted throughput of LTE/WiFi. Authors have introduced a weighting factor $\eta$ to balance the resources allocated to WiFi and LTE. For example, if the network operators are concerned about the WiFi throughput, by selecting a large value for $\eta$ more resources will be allocated to WiFi by increasing the LTE-off period. Similar to these works, this thesis has tried to increase the WiFi's performance by increasing it's transmission time by sending larger packets.

\section{$2.2 \quad$ WiFi Link adaptation}

Link adaptation literature can be categorized into two main groups. The first group aims to adapt the link via statistics of frame loss. In this approach the statistics of success and failure of previous transmission will be used in order to adapt the link for future transmission. These groups will increase the rate of transmission in the period of having a high successful transmission history and will decrease the bitrate when failures become frequent. However, the second approach will consider the received information as a base parameter for future link adaptation. In this group of algorithms, bit-rate will increase when the received signal has a high SNR and will decrease when signal strength is low. For both cases several important researches have been studied and are presented below.

\subsubsection{SNR based Link Adaptation}

The first study in the SNR based link adaptation group is Receiver Based Auto Rate (RBAR) [26]. The rate adaptation in this work is based on the signal strength. The authors have proposed that the receiver should evaluate the channel condition by measuring the Request to send (RTS) message sent by the transmitter. Receiver then set the data-rate transmission based on the analysis of the received RTS signal. This can be a good estimate of the channel since the transmissions are occurring shortly after the RTS signal. The RTS and Clear to Send (CTS) signals can also reduce the hidden node problem in a more crowded environment. Furthermore, Sadeghi et al [27] tries to enhance the performance of RBAR proposing the Opportunistic Auto Rate (OAR) algorithm. The key idea in this enhancement is to transmit multiple back to back packets when channel quality is high. OAR transmits multiple packets instead of one when the SNR indicates that a higher data-rate is possible. The number of 
packets transmitted in this way is in proportion with the achievable data rate over lowest rate ratio. Applying OAR results in assigning the same time to a sender as if was sending at the base rate, which leads to sufficient time for transmitting several packets according to the channel conditions. While RBAR and OAR may have good performances, they are not compatible with the 802.11 standard and implementing such algorithms requires some modification in the 802.11 standard [28].

The BEACON Auto Rate Adaptation (BARA) can be used [10] to determine the appropriate bit-rate especially when RTS/CTS exchange is disabled. The goal in this research is to use the transmitted beacon frames for estimating the channel conditions. Based on the received beacon frame information such as SNR and signal strength, sender determines the bit-rate for the transmission. Moreover they propose that a sending station listens to the transmitting packets in its environment to understand the channel conditions in real time. However, since beacon frames are transmitted with fairly long periods compared to the channel variation frequency they will not completely decode the channel status during the transmission..

\subsubsection{Frame loss based Link Adaptation}

The first link adaptation algorithm is Auto Rate Fallback (ARF) [29]. ARF tries to adapt to the changing conditions of the channel by using higher bit rates when the channel conditions permit. ARF records the number of successful transmission without any retransmission occurring in between. For initiating a transmission to a new device, it selects the highest bit rate. ARF then adjusts the bit-rate by the number of retransmission and packet acknowledgements. The algorithm moves to the next lowest bit-rate in the case of packet loss (no acknowledgement) and move to the next highest bit-rate after 10 consecutive successful transmissions. If none of these conditions occur it will continue with the current bit-rate transmission. The authors in [30] try to improve the performance of ARF by introducing Adaptive Auto Rate Fallback (AARF). They try to change the threshold of 10 successful transmissions continuously at run time in order to adapt to the channel conditions more efficiently. The adaptation in the threshold is based on a Binary Exponential Backoff (BEB) approach. If the transmission of a probing packet is unsuccessful, the number of continuous successful transmissions will be increased by a factor of two (reaching a maximum threshold of 50). The threshold is reset to 10 when the rate decreases due to two continuous failures in transmission. 
The authors in [31] proposed a Collision Aware Rate Adaptation (CARA) approach. The proposed algorithm tries to identify the collisions using two different methods, which are RTS probing and CCA. When there are two stations transmitting at the same time collision will happen leading to an unsuccessful transmission. Although, channel conditions are unaffected in these cases, ARF and AARF will reduce the transmission rate assuming the channel variation is responsible for the failure in transmission. This wrong assumption will result in a throughput degradation, since the channel conditions were not changed and the transmissions could have been followed up by a higher rate. Therefore, CARA tries to identify these cases and further enhance the performance by allowing the retransmissions to be continued with a higher rate. In this algorithm data frames are transmitted without the Ready To Send, Clear To Send RTS/CTS procedure. Only, in the case of an unsuccessful transmission RTS/CTS will be enabled. If a retransmission is successful, CARA will stay at the same rate and will transmit the next frame without RTS/CTS. This approach can further improve the ARF, and AARF methods.

The robust Rate Adaptation algorithm is proposed in [28]. In this approach, the loss ratio is estimated by keeping track of the frame loss in a short time window consisting of 5 to 40 frames. After that, the algorithm decides to increase, decrease, or use the same rate based on the calculated loss ratio. This algorithm also selectively turns on RTS/CTS procedure to reduce collision losses. Furthermore, the number of protected frames by the exchange of RTS/CTS will be adapted to the collision level by using Adaptive RTS (A-RTS). A-RTS employs the RTS window to keep track of frames needed to be transmitted with RTS enabled. A-RTS transmit the frames without RTS initially (RTS window $=0$ ), but if a collision happens the RTS window will be incremented by one and in the case of successful transmission the RTS window will be halved.

Onoe algorithm was introduced by the MadWiFi project [32]. This algorithm aims to find the best rate with a loss ratio below 50\%. For any individual link, Onoe will keep track of the number of credits that current bit-rate is achieving and if these credits pass over a threshold the bit-rate will be increased. The number of credits for an initial transmission will be set to zero. After that, the number of credits will be increased if less than one every ten packet need a retry and the credits will be decreased otherwise maintaining the lowest possible credit of zero. The rate adjustments will occur at the end of a one second period based on the collected 
transmission statistics. Therefore, it can be concluded that Onoe is not sensitive to the fast changes, incapable of adapting to the fast channel variation.

Sample Rate algorithm is proposed in [33]. Sample Rate tries the highest possible bit-rate for a starting link. If four consecutive unsuccessful transmissions happen for a bit-rate the usage of current bit-rate will be stopped. Every tenth packet, a sample bit-rate from the set of bit-rates higher than the current one will be selected for transmission. Moreover, Sample Rate calculates the transmission time for each bit-rate over a window size of 10 seconds. Based on the calculated statistics, the Sample Rate will adjust the bit-rate for the wireless link. A similar approach to Sample Rate is the Minstrel Algorithm [34]. However, while Sample Rate selects the rate with the lowest transmission time, Minstrel algorithm selects the rate with the highest throughput. In particular, Minstrel uses a multirate retry chain, consisting of four different rates (highest throughput, second highest throughput, highest probability success, lowest throughput) [35] using a fixed number of retry attempts for each rate. Similar to Sample Rate every 10th packet it sends a random rate to evaluate the transmission of the rest of the available rates. Every $0.1 \mathrm{sec}$ it performs a statistics calculation using an Exponential Weighting Moving Average (EWMA) method to take into considerations the statistics of previous $0.1 \mathrm{sec}$ intervals. Due to the efficiency of the Minstrel algorithm and its ability to adapt on the changing medium conditions, it has been selected as the Link adaptation algorithm for WiFi in this study.

\subsection{Link Adaptation in LTE}

Link adaptation in LTE is also similar with $\mathrm{WiFi}$, and involves selecting the modulation and coding scheme (MCS) to achieve the maximum data rate possible for each transmission to the UE [36]. However, in contrast with WiFi an adaptive modulation and coding (AMC) technology is used for LTE, enabling high spectral efficiency communication within the network [37]. In order to perform AMC operation in LTE, suitable feedback must be delivered from the UE back to the eNB.

This feedback is supplied through the Channel Quality Indicators (CQIs). The CQI value is an index that is correlated with the maximum throughput that can be achieved by properly choosing a pair of modulation and coding schemes meeting a target BLER Probability (e.g. 1\%, 10\%). Hence, link adaptation is put into effect by using a suitable CQI selection technique. The CQI is obtained by the UE with 


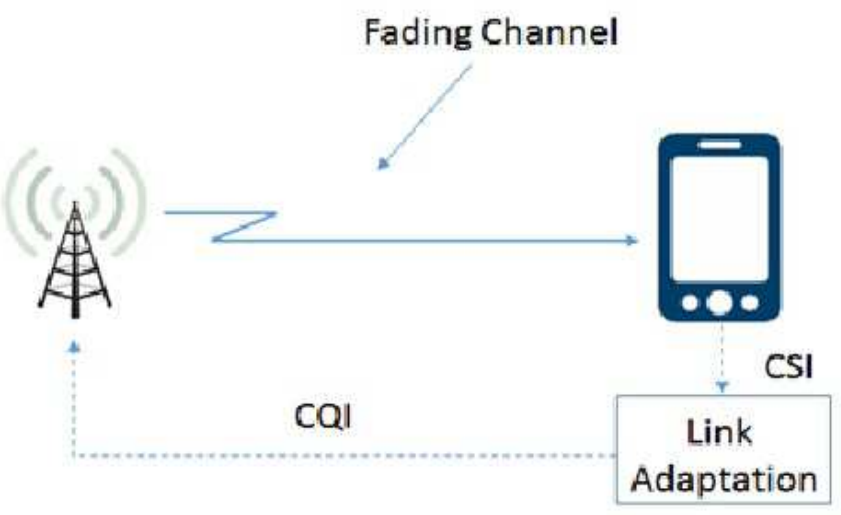

Figure 2.1: Link Adaptation in LTE Operation

respect to the Channel State Information (CSI) as depicted in Figure 2.1.

The general execution of the link adaptation mechanism for LTE is as follows [38] $:$

- Firstly, the noise variance and the channel matrix H should be extracted based on current channel condition.

- The post SINRs are computed for all subcarriers.

- With the SINRs it is possible to estimate a link quality metric to translate the CSI.

- The calculated metric is then mapped to a related reference BLER for the ongoing modulation and coding scheme utilizing the CQI search loop and a preexisting look-up table.

- The CQI search is established by repeating step (3) and (4) till the current CQI delivers BLER less than a threshold of a target BLER (e.g. 5\%).

- The chosen CQI will be reported back to the transmitter.

Effective SNR is the most frequent link quality metric. It is interpreted as the SNR observed by the codeword as if it were sent over a flat-fading channel [39]. A high SNR value results in a higher order modulation schemes having a higher spectral efficiency (e.g. 64QAM). On the contrary, with a low SNR value a lower order modulation scheme like QPSK will be used. 
Table 2.1: The 4-BIT CQI Table in LTE

\begin{tabular}{|c|c|c|c|}
\hline CQI Index & Modulation & Code Rate $\times 1024$ & Efficiency (bits/s/Hz) \\
\hline 0 Out of range 1 & QPSK & 78 & 0.1526 \\
\hline 2 & QPSK & 120 & 0.2344 \\
\hline 3 & QPSK & 193 & 0.3770 \\
\hline 4 & QPSK & 308 & 0.6016 \\
\hline 5 & QPSK & 449 & 0.8770 \\
\hline 6 & QPSK & 602 & 1.1758 \\
\hline 7 & 16QAM & 378 & 1.4766 \\
\hline 8 & 16QAM & 490 & 1.9141 \\
\hline 9 & 16QAM & 616 & 2.4063 \\
\hline 10 & 64QAM & 466 & 2.7305 \\
\hline 11 & 64QAM & 567 & 3.3223 \\
\hline 12 & 64QAM & 666 & 3.9023 \\
\hline 13 & 64QAM & 772 & 4.5234 \\
\hline 14 & 64QAM & 873 & 5.1152 \\
\hline 15 & 64QAM & 948 & 5.5547 \\
\hline
\end{tabular}

The SNR-BLER relationship in Additive White Guassian Noise (AWGN) channel can be extracted by simulation utilizing the modulation and coding schemes (MCS) determined in the CQI table as displayed in Table 2.1 (Table 7.2.3-1 TS 136213 V12.4.0 [40]).

Several link adaptation algorithms have been proposed for LTE. However, the Mutual Information approach [41], which follows the step above, presents the best performance. To this end, the MI algorithm has been selected in our simulations in order to compare the performance of the coexistence. In the simulation the Mutual Information approach (MI) which follows the steps above has been implemented as the LTE link adaptation technique. 


\section{Chapter 3}

\section{Theoretical Evaluations}

In this chapter, a theoretical evaluation of the WiFi throughput performance when operating alone and when coexists with LAA-LTE is presented. Furthermore, results and discussions for numerical analysis based on the theoretical evaluations are displayed.

\subsection{WiFi Performance without Interference}

Bianchi has presented a well-known analytical model for WiFi in [1]. In this study, the CSMA/CA procedure performed at the MAC layer has been modeled based on the Markov Chain concept. In the proposed model $n \mathrm{WiFi}$ stations are communicating in an ideal channel condition. Therefore, it can be concluded that any packet transmitted in this scenario will either be received successfully or will collide with another packet resulting in a transmission failure. All the stations are operating in a saturation mode condition meaning that after every transmission there is another packet in the queue (the probability of arriving packets in the queue, $q$, is 1 ). In the steady state every station will send a packet with probability $P_{t, w}$. For simplicity it has been assumed that in any given transmission attempt the collision probability seen by a transmitting station, $P_{c, w}$ or simply $P$ in Figure 3.1, is independent and

constant. $P_{c, w}$ can be calculated based on the fact, that in a time slot the probability of collision for a transmitting station is equal to the probability that one or more than one of the $n-1$ remaining stations transmit at the same time. Therefore:

$$
P_{c, w}=1-\left(1-P_{t, w}\right)^{n-1}
$$




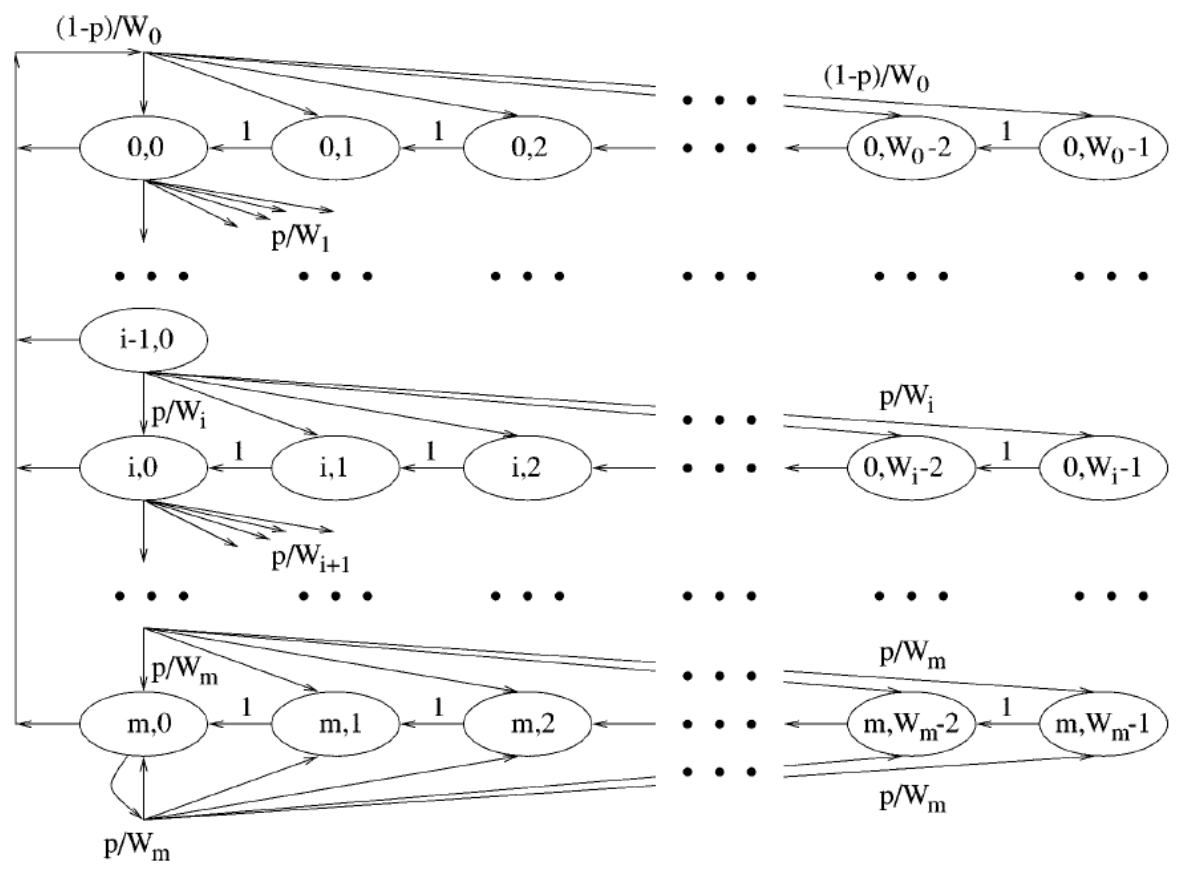

Figure 3.1: Markov chain model for the CSMA/CA [1]

and the probability of having at least one transmission in a slot time, $P_{t r, w}$, and having a successful transmission, $P_{s, w}$, in the under study network can be calculated through (2) and (3) respectively:

$$
\begin{gathered}
P_{t r, w, N}=1-\left(1-P_{t, w}\right)^{n} \\
P_{s, w, N}=n P_{t, w}\left(1-P_{t, w}\right)^{n-1}
\end{gathered}
$$

Also, the probability of collision in the overall network has been calculated in Bianchi's Markov chain Model in Figure 3.1 through the modeling of the back-off mechanism in CSMA/CA.

As it can be seen from this model, with every consecutive failure in transmissions the back-off window changes from $W_{i}$ to $W_{i+1}$ till the maximum back-off window $\left(W_{M}\right)$ is reached, where $W_{i+1}=2 W_{i}$. On the other hand, after a successful transmission the back-off window resets to the initial minimum value. The back-off counter also reduces by one after an idle time slot with probability 1 (the probability of channel being occupied, $P_{b}$ is zero in an idle time slot after the transmission). $P_{t, w}$ can be computed based on the probabilistic analysis of the chain in Bianchi's study through 


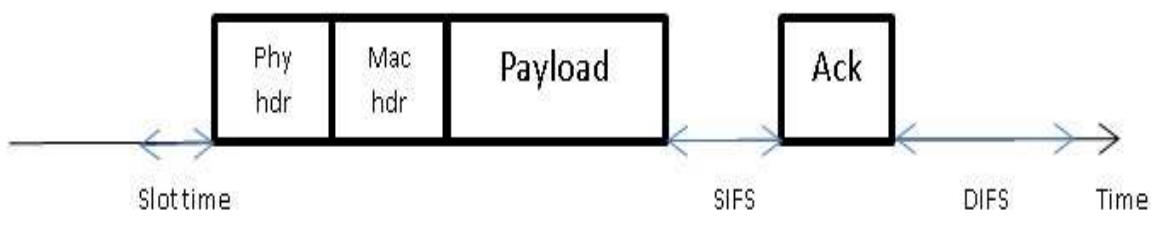

Figure 3.2: Time required for a successful transmission

(4):

$$
P_{t, w}=\frac{2\left(1-2 P_{c, w}\right)}{\left(1-2 P_{c, w}\right)(W+1)+P_{c, w} W\left(1-\left(2 P_{c, w}\right)^{m}\right)}
$$

where $W$ is the minimum window size, and $m$ is the maximum back-off stage. By solving (1) and (4), $P_{t, w}$ and $P_{c, w}$ can be derived numerically.

For computing the throughput for WiFi we need to find the expected payload bits transmitted over the expected required time period.

$$
S=\frac{E[\text { number of payload bits transmitted }]}{E[\text { time period }]}
$$

The average number of payload bits transmitted over the medium is $P_{s, w, N} E\left[L^{W i F i}\right]$, where $E\left[L^{W i F i}\right]$ is the length of transmitted packet excluding the header. The time duration for transmission consists of an empty time slot with the probability of (1 $\left.P_{t r, w, N}\right)$, the successful transmission period $\left(T_{s, w}\right)$ with probability $P_{s, w, N}$, and the collision period $\left(T_{c, w}\right)$ with probability of $P_{c, w, N}=P_{t r, w, N}-P_{s, w, N}$. Therefore, the achieved throughput can be calculated through (6):

$$
S_{w}=\frac{P_{s, w, N} E\left[L^{W i F i}\right]}{\left(1-P_{t r, w, N}\right) \delta+P_{s, w, N} T_{s, w}+P_{c, w, N} T_{c, w}}
$$

where $\delta$ is the time slot period. According to the Figures 3.2-3.3, $T_{c, w}$ and $T_{s, w}$ can be computed through (7) and (8):

$$
T_{s, w}=\frac{E[L]}{R_{w}}+S I F S+\frac{A c k}{R_{w}}+D I F S+\delta
$$




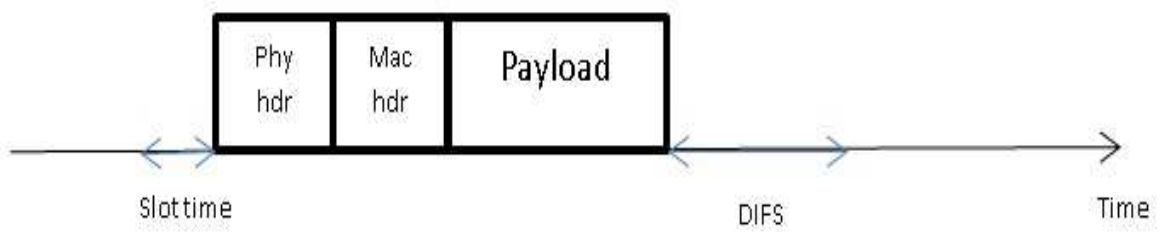

Figure 3.3: Time required for a collision

$$
T_{c, w}=\frac{E[L]}{R_{w}}+D I F S+\delta
$$

where $E[L]$ is the packet length including the header, $R_{w}$ is the transmission rate, $D I F S$ is the DCF interframe space, SIFS is the short interframe space, and Ack is the length of the acknowledgement frame.

\subsection{Coexistence Performance of WiFi and LAA}

In this part the performance of WiFi coexisting with LAA is analyzed. More specifically, the study focus on the medium access technique of both technologies which is CSMA/CA for WiFi and LBT for LAA. Among the different channel access schemes in LAA the thesis considers only the Category 4 (Cat4) [42] scheme which is more similar to WiFi CSMA/CA and therefore more compatible in the coexistence.

Cat4 LBT performs CCA in case of new transmission. If the channel is idle during CCA, LAA transmits immediately. Else, LAA enters in an extended CCA state (ECCA) starting with an initial contention window $\left(C W_{\text {min }}\right)$ of 32 . The $C W$ doubles each time reaching the maximum $C W\left(C W_{M}\right)$ size of 1024 when transmissions fail consecutively (like CSMA/CA procedure to reduce the collision probability). If another unsuccessful transmission happens after reaching the $C W_{M}$ the contention window will reset to $C W_{\min }$. LAA can transmit only after the random number that has been selected from $(0, C W)$, decrements toward reaching zero.

Based on the analysis of the Cat4 mechanism above, it can be concluded that it differs from CSMA/CA in two main parts. Firstly, CSMA/CA unlike Cat4 will not begin the transmission immediately after the CCA is successful (it has to select a random number and counts down to zero). Secondly, CSMA/CA does not restart to 


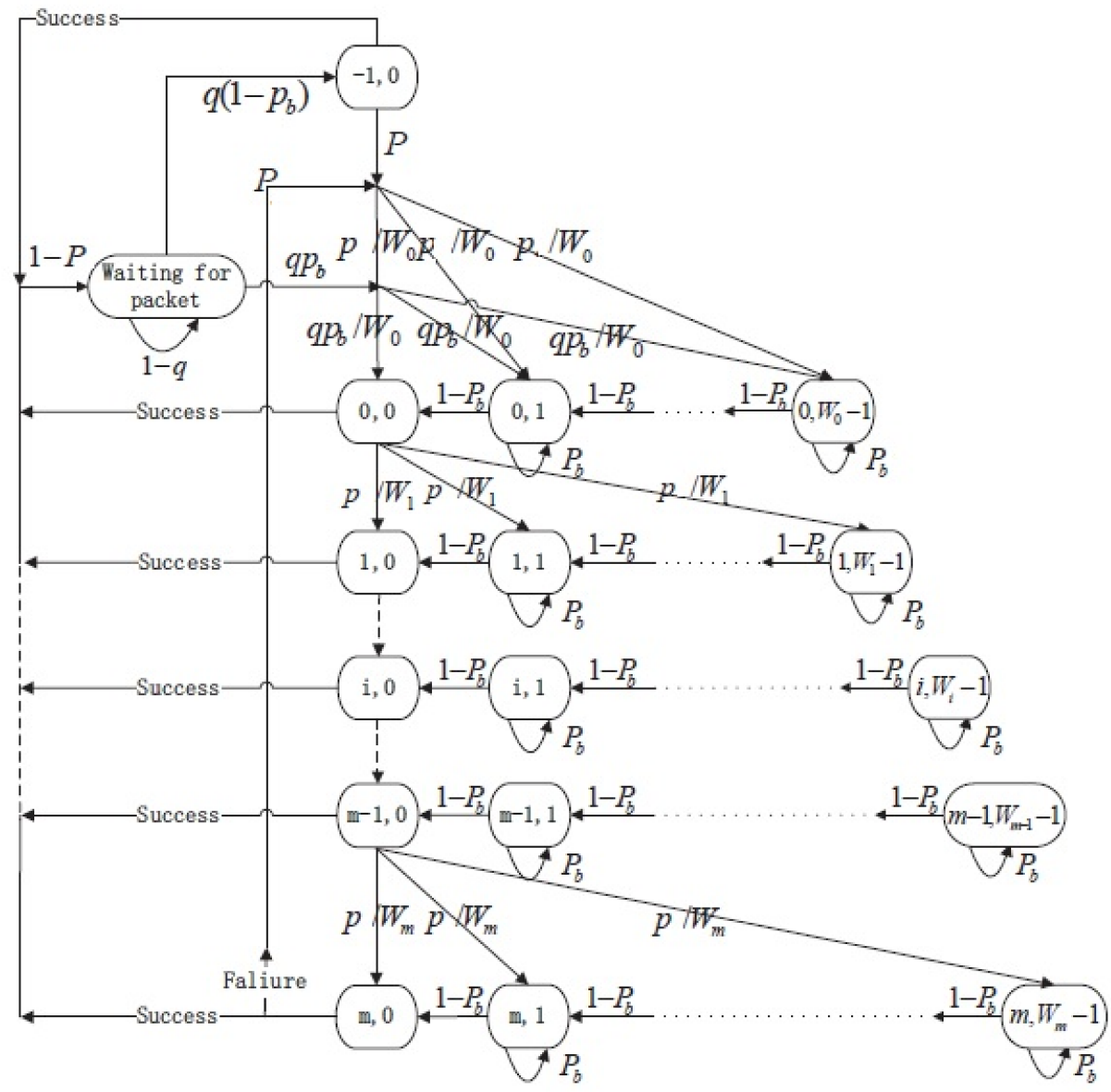

Figure 3.4: Markov chain model for the Cat4 LBT [2]

$C W_{\text {min }}$ from $C W_{M}$ after an unsuccessful transmission. CSMA/CA remains in the back-off state of choosing a random number from $\left(0, C W_{M}\right)$ interval (in the final state of back-off procedure) till a successful transmission happens. Except these two main differences these two medium access techniques almost operate in the same way to reduce the chance of collision.

\subsubsection{Analytical Evaluation for LBT}

An analytical model based on Markov chain for cat4 LBT coexisting with WiFi has been presented in [2]. In this model, $q$ represents the probability of packet arrival indicating that if $q=1$, the buffer is always full. $P_{b}$ is the probability of channel occupancy and $P_{c, L}(P$ in the Figure 3.4$)$ is the probability of failure in transmission 
due to collision. The state of LBT is displayed through $(s(t), z(t))$ where $s(t)$ represents the ECCA state and $z(t)$ denotes the counter. As it can be seen from the model in Figure 3.4 the LBT initially waits for the arrival of packets. When a packet arrives and CCA is successful with probability $q\left(1-P_{b}\right)$, LAA starts the transmission. Otherwise, LBT enters the ECCA state with an initial contention window of $W_{0}$. It can be concluded from the Figure 3.4 that LAA is only able to transmit when $z(t)=0, s(t) \in(-1,0, \ldots, m-1, m)$. Based on the analysis of this chain, authors have derived the probability of Cat4 transmission, $P_{t, L}$, as a function of probability of collision $\left(P_{c, L}\right)$ through $(9)$ :

$P_{t, L}=\frac{2\left(1-P_{c, L}\right)\left(1-2 P_{c, L}\right) C}{A+W B\left(1-P_{c, L}\right)\left(1-\left(2 P_{c, L}\right)^{m+1}\right)+B C\left(1-2 P_{c, L}\right)^{2}+2 C\left(1-P_{c, L}\right)^{3}\left(1-2 P_{c, L}\right)}$

where $A=2\left(1-P_{c, L}\right)^{2}\left(1-2 P_{c, L}\right), B=2 P_{c, L}-P_{c, L}^{2}, C=\left(1-P_{c, L}^{m+1}\right)$.

\subsubsection{Analytical Evaluation of Coexistence}

When there are i WiFi stations and j LAA Cat4 users (Figure 3.5 displays the under studied system model ) the probability of collision for a transmitting WiFi $\left(P_{c, w}\right)$ and LAA station $\left(P_{c, L}\right)$ are as follows:

$$
\begin{aligned}
& P_{c, w}=1-\left(1-P_{t, w}\right)^{i-1}\left(1-P_{t, L}\right)^{j} \\
& P_{c, L}=1-\left(1-P_{t, w}\right)^{i}\left(1-P_{t, L}\right)^{j-1}
\end{aligned}
$$

By solving (4),(9),(10), and (11) numerically, the probability of transmission and collision $\left(P_{t, w}, P_{c, w}, P_{t, L}, P_{c, L}\right)$ for WiFi and LAA can be extracted. Until the end of this chapter, all the remaining probabilistic analysis is based on the numerical computation of these four probabilities.

Intuitively, the probability of channel occupancy in time slots can be computed based on the fact that there should be at least one transmission in the channel and can be extracted through (12):

$$
P_{t r}=1-\left(1-P_{t, w}\right)^{i}\left(1-P_{t, L}\right)^{j}
$$

Channel occupancy probability for WiFi and LAA successful transmission can be 


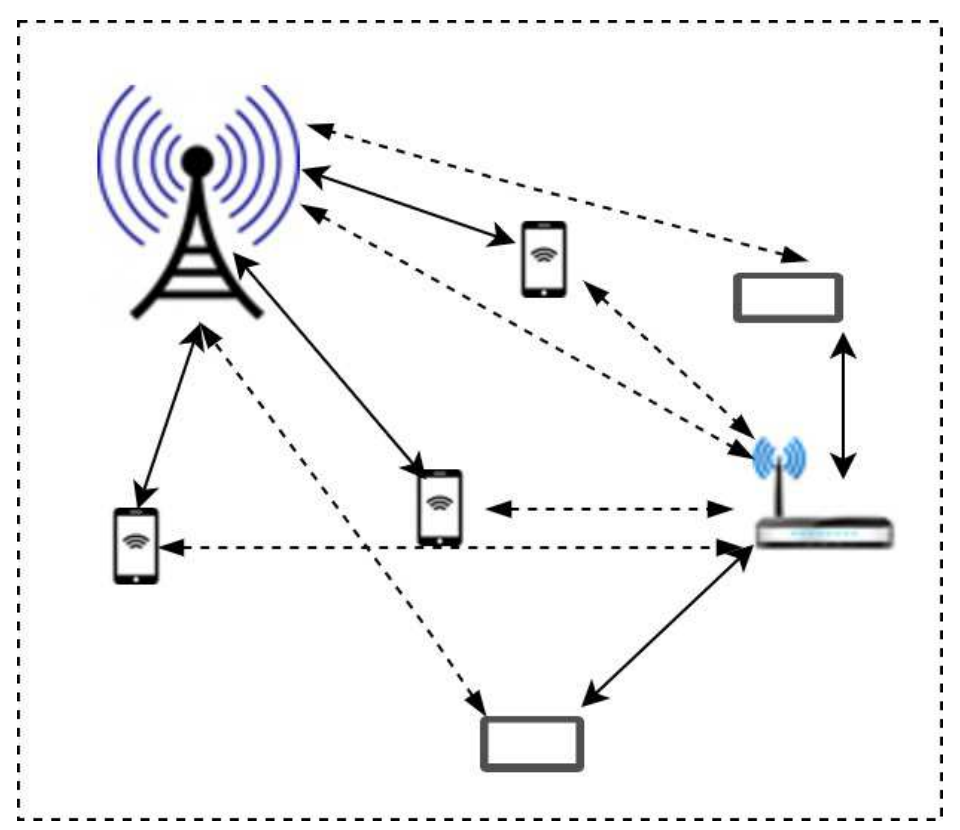

Figure 3.5: System model for the coexistence of WiFi and LTE. Solid connectors show the current attachments and dashed connectors display the potential connections.

calculated through the following equations respectively:

$$
\begin{aligned}
& P_{s, w, N}=i P_{t, w}\left(1-P_{t, w}\right)^{i-1}\left(1-P_{t, L}\right)^{j} \\
& P_{s, L, N}=j P_{t, L}\left(1-P_{t, L}\right)^{j-1}\left(1-P_{t, w}\right)^{i}
\end{aligned}
$$

In our specific model, the collision probability consists of three different events of: 1) WiFi unsuccessful transmission due to WiFi stations collision, 2) LAA failure because of LAA stations collision, and 3) collision between WiFi and LAA stations. The probability of these three events is derived below respectively [2]:

$$
\begin{gathered}
P_{c, w, N}=\left(1-\left(1-P_{t, w}\right)^{i}-i P_{t, w}\left(1-P_{t, w}\right)^{i-1}\right)\left(1-P_{t, L}\right)^{j} \\
P_{c, L, N}=\left(1-\left(1-P_{t, L}\right)^{j}-j P_{t, L}\left(1-P_{t, L}\right)^{j-1}\right)\left(1-P_{t, w}\right)^{i} \\
P_{c, w L, N}=P_{t r}-P_{s, w, N}-P_{c, w, N}-P_{s, L, N}-P_{c, L, N}
\end{gathered}
$$

For computing the achievable throughput for WiFi and LAA, we have to follow the same logic of equation (5) and (6). The only difference now is that the time interval for a successful transmission has to include three more time durations beside the previously mentioned ones. These three new periods are the time duration for 
successful transmission of LAA $\left(T_{s, L}\right)$ with probability of $P_{s, L, N}$, the time duration of collision within two LAA stations $\left(T_{c, L}\right)$ with probability $P_{c, L, N}$, and the period for a collision among WiFi and LAA, $\max \left(T_{c, L}, T_{c, w}\right)$, with probability $P_{c, w L, N}$. Therefore, the achievable throughput for WiFi and LAA can be derived from [2] :

$$
\begin{aligned}
& S_{w}=\frac{P_{s, w, N} E\left[L^{W i F i}\right]}{\left(1-P_{t r}\right) \delta+P_{s, w, N} T_{s, w}+P_{s, L, N} T_{s, L}+P_{c, w, N} T_{c, w}+P_{c, L, N} T_{c, L}+P_{c, w L, N} \max \left(T_{c, w}, T_{c, L}\right)} \\
& S_{L}=\frac{P_{s, L, N} E\left[L^{L A A}\right]}{\left(1-P_{t r}\right) \delta+P_{s, w, N} T_{s, w}+P_{s, L, N} T_{s, L}+P_{c, w, N} T_{c, w}+P_{c, L, N} T_{c, L}+P_{c, w L, N} \max \left(T_{c, w}, T_{c, L}\right)}
\end{aligned}
$$

where $T_{s, L}, T_{c, L}$ are the time duration required for successful and unsuccessful transmissions in LBT and can be found from the following equations:

$$
\begin{gathered}
T_{s, L}=\frac{E[L]}{R_{L}}+\frac{A c k}{R_{L}}+D I F S+\delta \\
T_{c, L}=\frac{E[L]}{R_{L}}+\frac{N a c k}{R_{L}}+D I F S+\delta
\end{gathered}
$$

\subsection{Numerical Analysis of WiFi and LAA Coexis- tence}

In this part the numerical results of different combination of WiFi and LAA users is shown in order to validate the operation of the back-off stage mechanism of the two networks. Specifically, the results for the transmission and collision probability of WiFi and LAA stations are presented. For finding the transmission and collision probabilities we solved the (4),(9),(10), and (11) numerically for different combination of number of users in the two technologies.

Figure 3.6 illustrates the transmission probability of one WiFi station (blue line). Since WiFi will choose a back-off window size of 32 the transmission probability of one WiFi station in an empty time slot is low. Based on the calculated numbers it can be decided that WiFi stations are mainly operating in the first and second back-off stage. The average transmission probability of a WiFi station operating in the first back-off stage is 0.0625 since the window size is $32\left(P_{t, w}=1 / 16=0.0625\right)$. The average probability is calculated under the assumption that each station belonging to WiFi or LAA has the same probability of transmission and face the same probability 


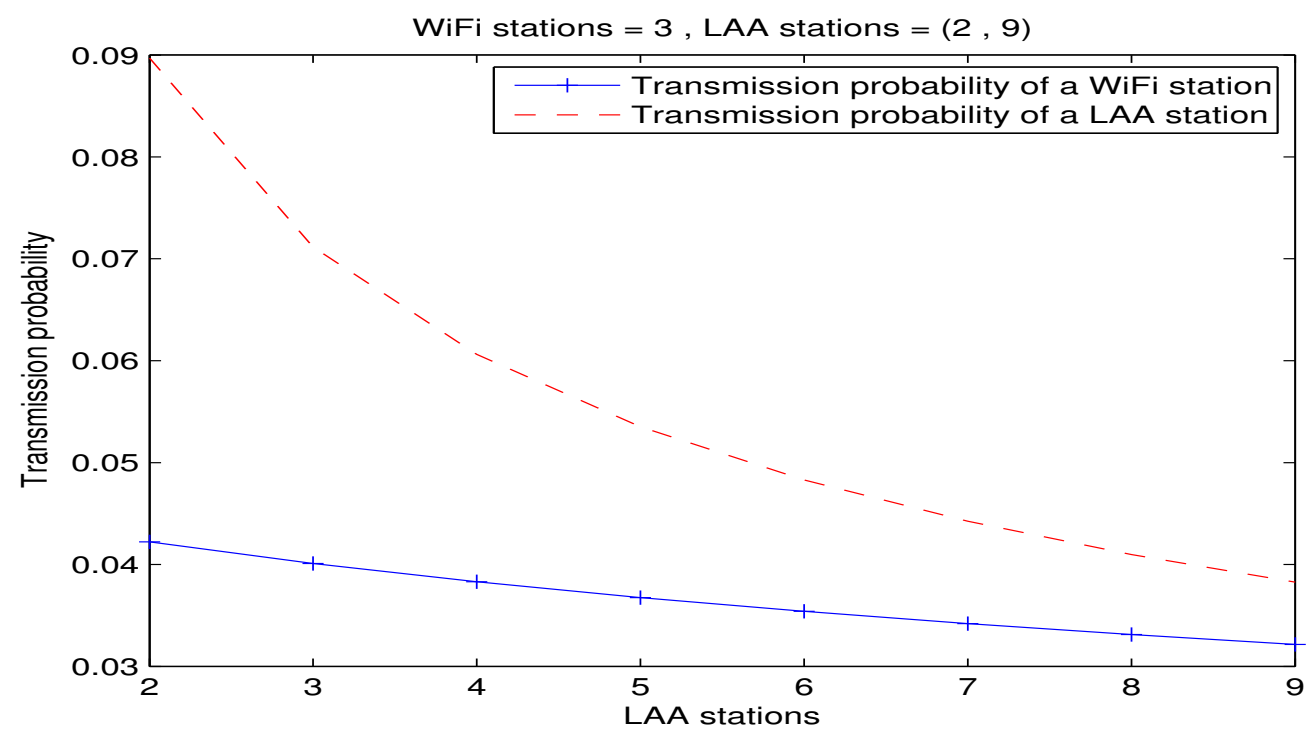

Figure 3.6: Comparison of transmission probability of WiFi and LAA

of collision.

On the other hand, the transmission probability of one LAA station can be viewed in Figure 3.6 (red line) indicating LAA stations in average operate in the back-off stage of two and three. In the back-off stage one, window size is zero indicating that LAA can transmit after an empty DIFS period. Therefore, it can be seen that the LAA probability of transmission is larger than the WiFi transmission probability. Furthermore, when the number of stations increases in the system the transmission probability of WiFi and LAA get closer to each other.

Figure 3.7 shows the collision probability seen by a transmitting WiFi and LAA stations (it is assumed that the station is ready to transmit and it is not waiting for any back-off). As it appears, the probability of collision seen by WiFi stations is larger than LAA, since the LAA approach is more aggressive for grabbing the channel. When the number of LAA stations are low (two LAA stations and three WiFi stations), the probability of collision for a transmitting LAA station is $20 \%$ less than the probability of collision of a WiFi station. But as the number of LAA stations increases the difference in the collision probability seen by a transmitting WiFi or LAA station becomes much smaller and around $1 \%$. The reason can be explained through analyzing the beginning and end of the $\mathrm{x}$ axis. At the beginning, when the number of LAA stations is two any given LAA station ready to transmit sees one LAA and three WiFi Stations and a WiFi station sees two WiFi and two 


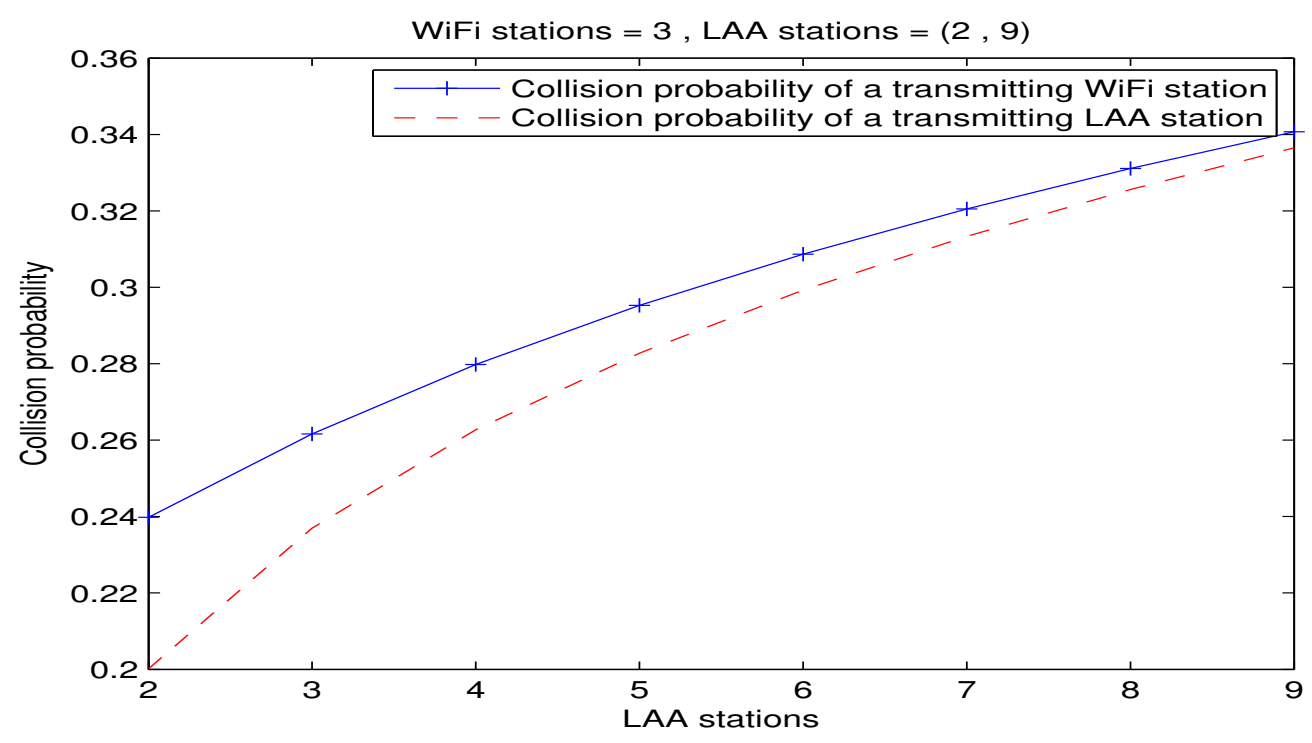

Figure 3.7: Comparison of collision probability of WiFi and LAA

LAA stations in the network. But at the end, when the number of LAA stations is nine a transmitting LAA station sees eight LAA and three WiFi and a WiFi stations sees two WiFi and nine LAA. It is apparent that the difference in the second case within the collision probability of $\mathrm{WiFi}$ and LAA stations is much smaller than the first case.

In the Table 3.1 the transmission and collision probability of LAA and WiFi is shown for different combination of number of stations in each network within $(2,5)$ for WiFi and $(2,9)$ for LAA. We can draw a conclusion that same behavior goes for the different number of stations of WiFi. Also the transmission probability of WiFi and LAA reduces by the increase in the number of WiFi stations. On the other hand, the collision probability increases by the increase in WiFi stations.

\subsubsection{Performance Analysis for Fair Coexistence of WiFi and LTE-LAA}

In this part, our goal is to make the coexistence of WiFi and LAA fair through changing the maximum packet size in the WiFi network as our first contribution in this work (since LAA employs fixed blocks of frequency-time in it's physical layer, the packet length for LAA cannot be adapted easily for having a fair coexistence of 
Table 3.1: Transmission and collision probability of WiFi and LAA for different number of stations in each network

\begin{tabular}{c|c|c|c|c|c} 
WiFi Stations & LAA Stations & $P_{t, w}$ & $P_{t, L}$ & $P_{c, w}$ & $P_{c, L}$ \\
\hline \hline 2 & $2-5$ & $0.038-0.043$ & $0.058-0.103$ & $0.230-0.286$ & $0.179-0.270$ \\
\hline 2 & $6-9$ & $0.033-0.036$ & $0.040-0.052$ & $0.300-0.333$ & $0.288-0.328$ \\
\hline 3 & $2-5$ & $0.037-0.042$ & $0.054-0.090$ & $0.240-0.295$ & $0.200-0.283$ \\
\hline 3 & $6-9$ & $0.032-0.035$ & $0.038-0.048$ & $0.309-0.341$ & $0.299-0.334$ \\
\hline 4 & $2-5$ & $0.036-0.041$ & $0.050-0.079$ & $0.252-0.305$ & $0.221-0.295$ \\
\hline 4 & $6-9$ & $0.031-0.035$ & $0.036-0.045$ & $0.318-0.348$ & $0.310-0.345$ \\
\hline 5 & $2-5$ & $0.035-0.040$ & $0.046-0.070$ & $0.264-0.315$ & $0.241-0.307$ \\
\hline 5 & $6-9$ & $0.031-0.034$ & $0.034-0.042$ & $0.327-0.356$ & $0.321-0.353$
\end{tabular}

WiFi and LAA). Although the definition of fairness in the coexistence of WiFi and LTE is not fixed yet [43], several $3 \mathrm{GPP}$ members believe that fair access means that WiFi and LAA should receive equal level of throughput [44]- [43]. Nevertheless, a fair access could also mean that the LTE-LAA and the WiFi should achieve an equal throughput per number of stations exist in each. Both of these two definition of fairness have been considered in this work.

Figure 3.8 shows the ratio of WiFi packet payload over LAA packet payload in order to achieve an equal throughput for both Radio Access Networks (RANs). In order to extract the WiFi/LAA frame independent of the number of users in the system we have assumed that the number of WiFi and LAA stations are equally distributed. More specifically, achieving an equal throughput can be possible by adding an extra constraint, $P_{s, w, N} E\left[L^{W i F i}\right]-P_{s, L, N} E\left[L^{L A A}\right]=0$, to the calculated $P_{s, w, N}$ and $P_{s, L, N}$ (these two probabilities are computed based on the $P_{t, w}, P_{c, w}, P_{t, L}, P_{c, L}$ which had been extracted numerically). As it can be seen from Figure 3.8 the ratio of $\mathrm{WiFi} / \mathrm{LAA}$ frame becomes smaller and close to 1 by increasing the number of WiFi and LAA stations. This is due to the fact that with the increase of the number of incumbents in the channel, the difference in the achievable throughput for both WiFi and LAA stations becomes smaller. 


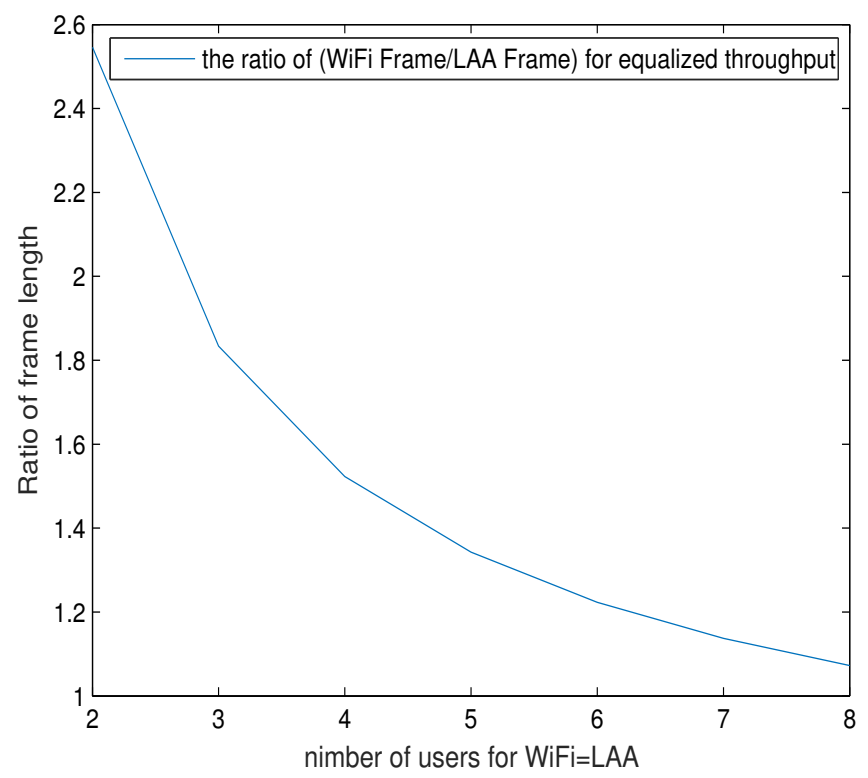

Figure 3.8: Packet length ratio of WiFi over LAA for having equal throughput. The number of users for both networks are the same.

The performance gap can be calculated by $\frac{\text { WiFi throughput }}{L A A \text { throughput }}$, which is a function of $\frac{P_{s, w, N}}{P_{s, L, N}}$ and $\frac{\text { WiFiFrame }}{\text { LAAFrame }}$. The $P_{s, L, N}, P_{s, w, N}$ probabilities do not only depend on MAC layer parameters, such as minimum and maximum back-off window sizes, but also depends on the number of WiFi and LAA stations.

Previously, we found the WiFi/LAA frame ratio by doing a numerical analysis. But there can be another approach that we can skip the finding for an exact value for WiFi Frame in order to have same throughput with LAA (Fairness criterion: equal WiFi and LAA throughput). Instead, a heuristic approach for calculating a suboptimal WiFi/LAA frame ratio can be utilized based on $\frac{\text { Number of WiFi stations }}{\text { Number of LAA stations }}$. In detail, we introduce two parameters $c$ and $\alpha$ to compute the WiFi Frame in the following way:

$$
\frac{\text { WiFiFrame }}{\text { LAAFrame }}=c \times\left(\frac{\text { Number of WiFi stations }}{\text { Number of LAA stations }}\right)^{\alpha}
$$

In this equation $c$ compensates for the performance gap between one WiFi and one LAA station. $\alpha$ on the other hand, compensates the different ratio of number of users in WiFi and LTE. After that, $c$ and $\alpha$ are appropriately fine-tuned to minimize 


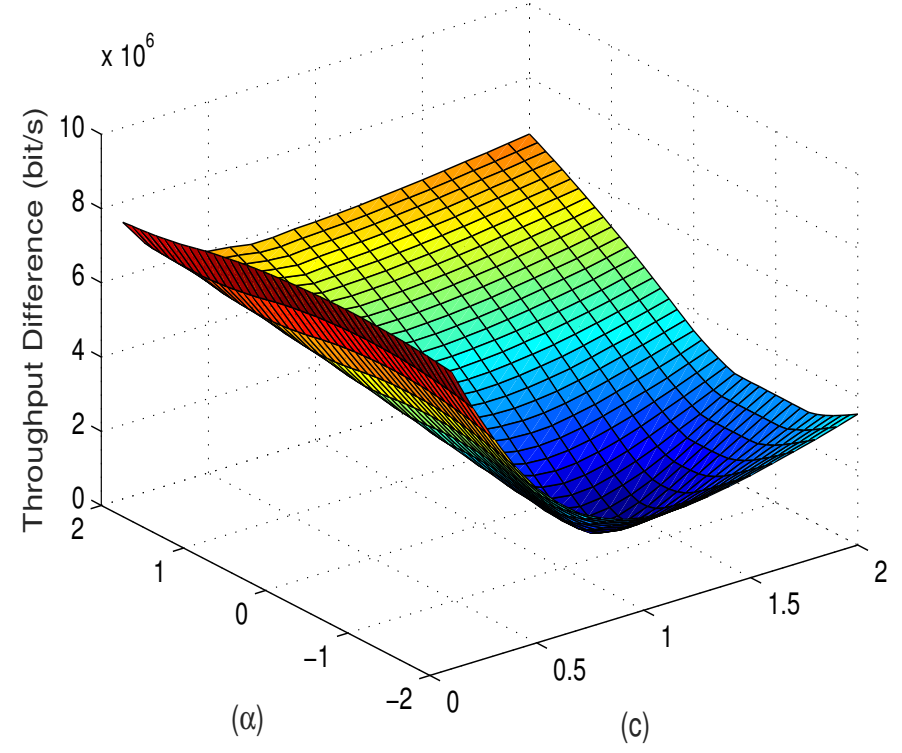

Figure 3.9: Average throughput difference of WiFi and LAA for different numbers of $\alpha$ within $(-2,2)$ and $c$ within $(0,2)$. Fairness criterion: WiFi throughput $=$ LAA throughput

the throughput gap between these two networks. Figure 3.9 displays the throughput difference for different values of $\alpha$ within the range of $(-2,2)$ and $c$ within the range of $(0,2)$. For all of the combinations of $c$ and $\alpha$, the throughput difference is calculated, based on averaging over different selection of number of stations for WiFi and LAA. For example, if $\alpha=c=0.1$, the difference in throughput is the average for 81 cases of having $a$ and $b$ number of stations in WiFi and LAA respectively, where $a, b \in(1,2, \ldots, 9)$. The reason for this selection is that in our analysis we did not consider the effect of hidden node problem. Hidden node in larger networks can play a much important role when the number of competing stations are large leading to a far less accurate results. This averaging can provide a good adaptability of $c$ and $\alpha$ in different crowded situations. It can be seen from the Figure 3.9 that the throughput difference is a convex surface and there exists a local optimum value for $c$ and $\alpha$ to achieve the minimum difference in throughput. In this case $c$ and $\alpha$ have been found to be 1.2 and -1 respectively.

Figure 3.10 shows the ratio of LAA throughput over the WiFi throughput for different number of $\mathrm{WiFi}$ stations. In order to accommodate different cases we have 


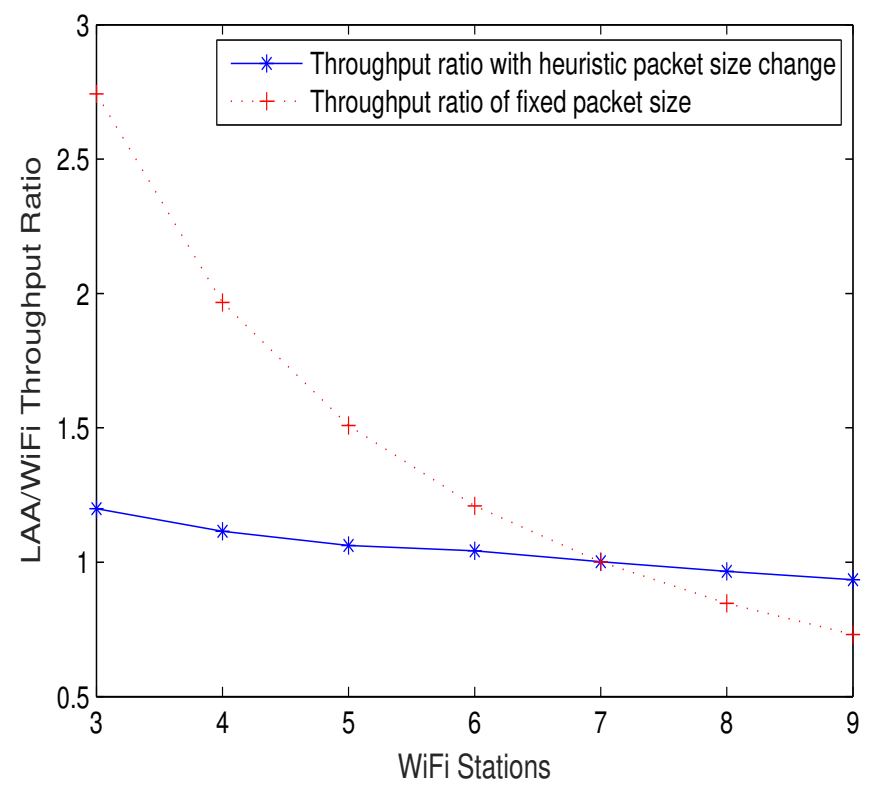

Figure 3.10: LAA/WiFi average throughput ratio for changing packet size based on heuristic approach versus the fixed packet size (LAA users are varying from 3 to 9 and the ratio is the average of all cases). Fairness criterion: WiFi throughput $=$ LAA throughput

considered the average ratio for varying LAA stations in case that $b \in(1,2, \ldots, 9)$ (In average there are 5 LAA stations). Figure 3.10 displays the comparison of our heuristic approach with the case that there is no packet size change for the WiFi network. As we can see the throughput ratio for the heuristic approach is close to 1 for all tested combinations. However, for the fixed packet size scenario the throughput ratio exceeds 1 for a small number of coexisting WiFi stations. This is due to the fact that besides LAA outperforming WiFi, when the number of WiFi stations are low, average number of LAA stations is larger than that of WiFi and it goes below one for a large number of WiFi stations. On the other hand, when the WiFi stations outnumber the average LAA stations (WiFi stations $=9$ ) the ratio goes below 1 .

The other fairness criterion in the context of coexistence of WiFi and LAA is that LAA and WiFi should receive the same amount of throughput per number of stations exist in each. The same approach like what we previously employed, can be utilized to achieve minimum difference in throughput per number of stations for WiFi and LAA. This time Figure 3.11 shows the different combinations of $c$ and $\alpha$ to achieve the minimum throughput difference per number of stations for the two 


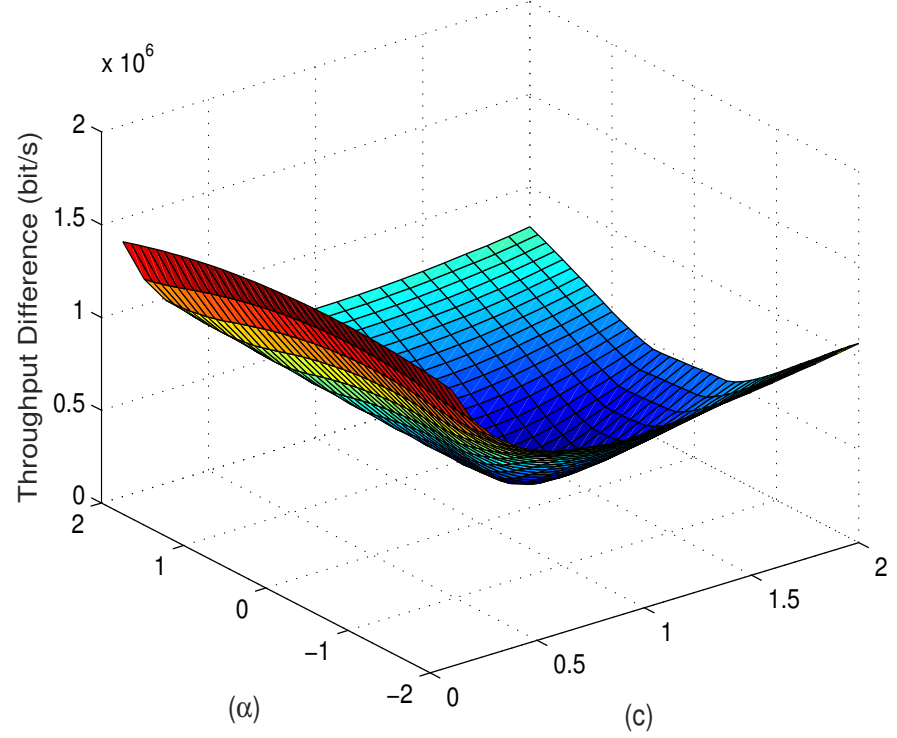

Figure 3.11: Average throughput difference of WiFi and LAA for different numbers of $\alpha$ within $(-2,2)$ and $c$ within $(0,2)$. Fairness criterion: WiFi throughput per number of station $=$ LAA throughput per number of station

technologies. Comparing with the Figure 3.9, we can observe that the throughput difference is significantly lower for the Figure 3.11. Such behavior can be explained through the absence of unbalanced number of WiFi and LAA stations in the shared channel. For the latter fairness criterion the optimum value for $c$ and $\alpha$ have been found to be 1.3 and 0 respectively. Optimum alpha being zero means that now the $\mathrm{WiFi}$ LAA frame ratio is independent of the number of stations in each RAN. This is a reasonable finding since we have already considered the effect of number of stations in each network.

Figure 3.12 depicts the ratio of LAA/WiFi throughput for the fairness criterion stated above. Like the Figure 3.10 the ratio of LAA/WiFi throughput is the average of varying LAA users to cover different scenarios. The proposed heuristic approach outperforms the fixed packet size in terms of fairness. However, the difference is not that large compared to the previous Figure 3.10 due to the nonexistence of unbalanced number of stations in the two RANs. 


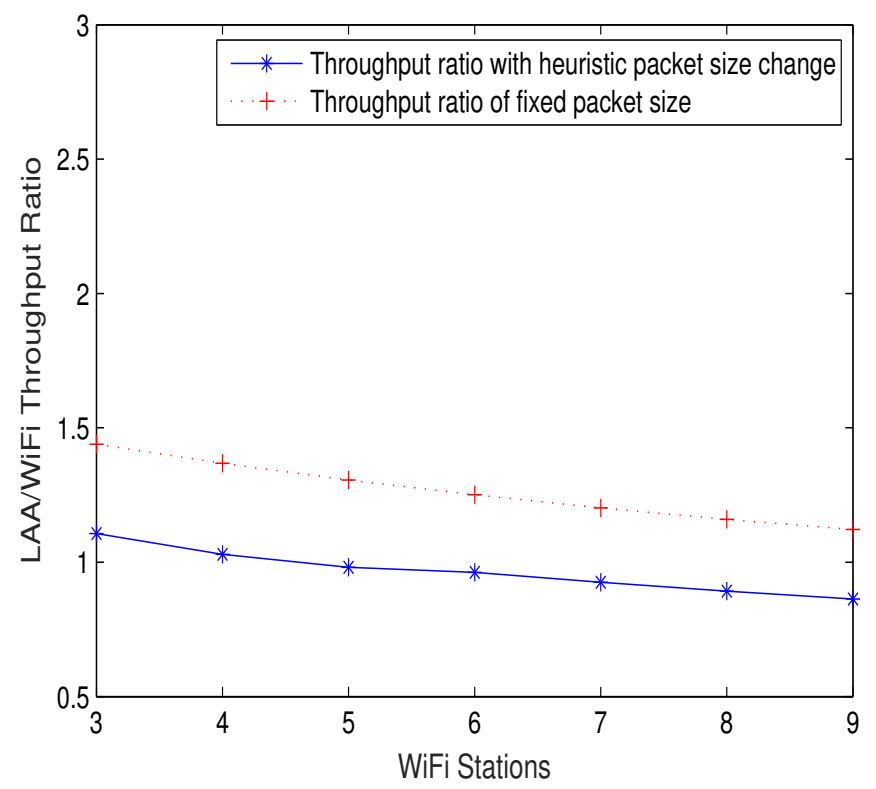

Figure 3.12: LAA/WiFi average throughput ratio for changing packet size based on heuristic approach versus the fixed packet size (LAA users are varying from 3 to 9 and the ratio is the average of all cases). Fairness criterion: WiFi throughput per number of station $=$ LAA throughput per number of station

\subsection{Allocation of Users between the two RANs}

In the last part of this chapter, we are investigating the allocation of users within WiFi and LAA in the shared channel as our second contribution in this thesis. To serve this purpose, we compare the following three different methods in the Figure 3.13. Figure 3.13 illustrates the comparison of throughput difference between WiFi and LTE for the following three approaches. In all of these methods users will arrive and depart according to a Poisson distribution. In order for all the users to maintain a minimum achievable throughput (depending on which rate we are using) a maximum number of users allowed in the system is defined (in our case maximum number is 20 users).

- In the first approach users will be allocated randomly to WiFi and LAA network.

- In the second one users will be allocated step by step with regards to minimizing the throughput difference of both networks.

- Finally, in the third approach, not only new users will be allocated step by 
Table 3.2: Simulation Parameters

\begin{tabular}{c|c} 
Parameter & Value \\
\hline \hline Channel Bandwidth & $20 \mathrm{MHz}$ \\
\hline Slot Duration & $9 \mu \mathrm{s}$ \\
\hline DIFS & $34 \mu \mathrm{s}$ \\
\hline SIFS & $16 \mu \mathrm{s}$ \\
\hline Rate & 1 QPSK, code rate $(1 / 2))$ \\
\hline Packet Payload & $10000 \mathrm{bits}$ \\
\hline Packet Header & 416 bits \\
\hline Poisson Arrival Rate & $2 /$ hour \\
\hline Poisson Departure Rate & $0.33 /$ hour \\
\hline Ack Length & 304 bits
\end{tabular}

step but also users departing the network will be relocated to reduce the gap between WiFi and LAA performance. This means that with a departure from WiFi or LAA the overall system can switch one of the users from WiFi or LAA to the counter network for fairness considerations.

We have assumed that the two RANs can exchange information through control signaling to divide the users among them. As it can be seen from the Figure 3.13, dynamically arranging the users based on both the arrivals and departures can significantly reduce the performance gap within the two networks (the fairness criterion considered in this case is the equal WiFi and LAA throughput. With the other fairness criterion also similar behavior can be observed). Table 3.2 shows the simulation parameters considered in the described scenario. 


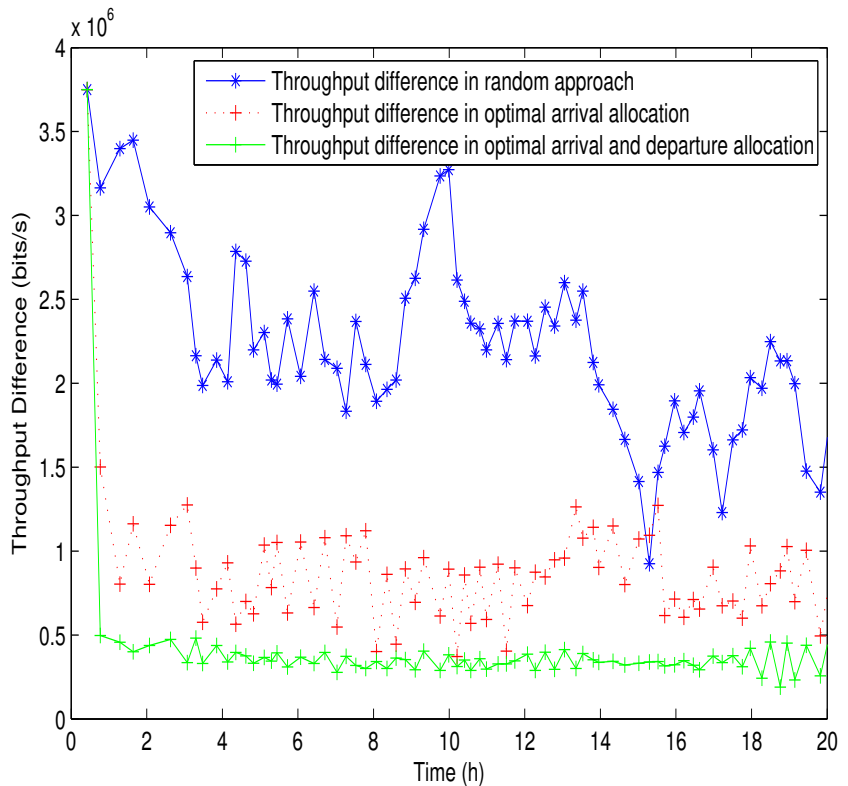

Figure 3.13: Comparison of three approaches: i)Random allocation of users, ii)Optimal allocation of users upon arrivals, iii)Optimal allocation of users upon both arrivals and departures 


\section{Chapter 4}

\section{Link Adaptation Considerations}

In the previous chapter we analyzed the throughput performance achieved in coexistence considering a static and identical rate for both RANs. However, in a realistic environment appropriate link adaptation algorithms are applied that allow to better utilize the available spectrum. To this end, in this chapter, we analyze the link adaptation mechanism applied in WiFi and propose a number of enhancements towards reducing the performance gap with the LTE. Specifically, the Minstrel algorithm is selected as the link adaptation approach in WiFi, due to it's widely usage in wireless drivers such as Madwifi, Ath5, Ath9 [45]. Minstrel also shows good performance compared to the other link adaptation techniques [46]. Hence, an enhanced Minstrel algorithm is proposed to better interpret the conditions of the coexistence in the wireless medium. Furthermore, the Mutual Information algorithm, the LTE link adaption technique, will be discussed.

\subsection{Overview of Matlab Simulator}

The simulator used is an event based simulator written in Matlab and can support experimentations in the IEEE 802.11 and the coexistence of WiFi with LAA. The main functionalities of $\mathrm{WiFi}$ and LAA supported by the simulator are as follows:

\section{WiFi:}

- Physical Layer:

- Most aspects of the 802.11ac specifications are already implemented.

- The full set of 802.11 TGn channel models from A to F can be selected 
- MAC and Higher Layers:

- Simulations involve one AP

- Two traffic models are supported: i) full buffer, ii) simple Poisson model (i.e. packets/second).

- A CSMA/CA algorithm with binary exponentially back off is implemented.

\section{LAA:}

- Physical Layer:

- A detailed SINR computation per subcarrier is used.

- MAC and Higher Layers:

- A Listen Before Talk (LBT) algorithm is implemented with binary exponential backoff.

The simulator follows a simple event sequence to drive the whole simulation. The events represent a specific action that needs to be performed by PHY and MAC layers. Each involved device needs to take appropriate actions when an event is raised. An example is when an event is triggered to schedule the start or stop of a transmission. Furthermore, according to the event triggered each device updates an internal state regarding its status (e.g. transmit, receive, idle, etc.).

For the execution of the simulator a main class needs to be run. The main class gets as input two configuration files one for the WiFi and one for the LAA. Otherwise, the WiFi configuration file can be the only input. These configurations files contain the necessary parameters that need to be set for the two technologies. Following, we refer the main parameters configured for WiFi and LAA:

\section{WiFi configuration parameters:}

- Number of Stations (STAs)

- Distance between AP and STAs 
- Channel bandwidth

- Guard interval

- Channel parameters (e.g. TGn channel modes, speed of the moving device)

- Traffic model for the uplink and downlink

- Activation of Link adaptation or using a fixed Modulation and Coding scheme (MCS)

- Transmission power

- Packet length

\section{LAA configuration parameters:}

- Number of user equipments (UE)

- Packet Length

- Offset distance between AP and BS

- Distance between UE and BS

- Number of subframes in uplink and downlink

- Traffic model for the uplink and downlink

- Activation of Link adaptation or using a fixed MCS

- Speed of the moving device

The main class parse the configuration files and set the experimentation environment accordingly.It checks if only the WiFi configuration file is provided and creates an environment with a single AP, otherwise it proceeds with the creation of both WiFi and LAA. Following the channel is created according to the information provided by the configuration files. Moreover, the set of the events that need to be triggered are also generated (e.g. packet generation, transmission duration, etc.). Then the actual simulation takes place. While executing the simulation, the events are triggered and the necessary actions are initiated from the involved devices. 


\subsection{Original Minstrel Algorithm}

Minstrel algorithm has been developed by the Madwifi project [34]. The theory behind the Minstrel Algorithm is that the algorithm can select the optimum rate by statistically calculating which bit-rate will result in the best throughput. Minstrel's operation can be classified into three main parts: I) Retry Chain, II) Rate Selection and III) Statistics Computation.

\subsubsection{Retry Chain}

Retry chain allows Minstrel to adapt to current channel variations. Specifically, retry chain consists of a table with four rate/count pairs. Minstrel will first send the packet with rate $R_{1}$ retransmitting $C_{1}$ times if the packet is lost. After $C_{1}$ unsuccessful retransmissions the second rate $R_{2}$ will be used for $C_{2}$ times and so on until all four pairs are tried. Rates $R_{1}$ and $R_{2}$ are set with the MCS indexes that can achieve the highest and second highest expected throughput. $R_{3}$ uses the rate with the highest success probability and $R_{4}$ is the base rate.

\subsubsection{Rate Selection}

The transmission during the Minstrel implementation are categorized into two different cases: 1) $90 \%$ of the transmissions are following a normal mode, where packets are sent according to the four rate/count pairs in the retry chain 2) 10\% of the transmissions follow a sampling mode, where a sample rate from the existing rates (excluding the four rates already being in the retry chain) is selected randomly. Minstrel also initialize the transmission based on a random rate. The goal of this approach is to gather statistics about the achieved throughput and probability of success for the rest of the algorithms. Table 4.1, provides information of how retry chain table changes according to the current transmission mode and if the sample rate is higher than the highest throughput in the retry chain or not.

\subsubsection{Statistics Calculation}

Every 100ms Minstrel updates the probability of success and the effective throughput for every rate. To do so it employs an Exponentially Weighted Moving Average 
Table 4.1: Minstrel Retry Chain (T: highest throughput, t: second highest throughput, P: highest probability, B: Base Rate, S: sample rate)

\begin{tabular}{c|c|c|c|c} 
Retry Index & 1 & 2 & 3 & 4 \\
\hline \hline No Sampling & $\mathrm{T}$ & $\mathrm{t}$ & $\mathrm{P}$ & $\mathrm{B}$ \\
\hline Sample Higher & $\mathrm{S}$ & $\mathrm{T}$ & $\mathrm{t}$ & $\mathrm{P}$ \\
\hline Sample Lower & $\mathrm{T}$ & $\mathrm{S}$ & $\mathrm{t}$ & $\mathrm{P}$ \\
\hline
\end{tabular}

(EWMA) approach to calculate the necessary statistics and update the retry chain. In detail, first the probability of success for the $100 \mathrm{~ms}$ period for every rate is calculated by:

$$
P=\frac{\text { number of successful transmissions }}{\text { total number of transmissions }}
$$

afterwards, the EWMA probability is calculated as follows:

$$
P_{E W M A}=P_{E W M A} \times \alpha+P \times(1-\alpha)
$$

So the success probability is updated every $100 \mathrm{~ms}$ taking into consideration both the success probability of the current $100 \mathrm{~ms}$ interval as well as the past intervals, using the EWMA factor $\alpha$, which denotes the weight that is given to the recent probability of success $P$. The value of $\alpha$ is set equal to 0.75 . Finally, the effective throughput is calculated using the flowing formula:

$$
S_{w}=P_{E W M A} \times \frac{\text { Number of transmitted bits }}{100 \mathrm{~ms}}
$$

According to the new values of the effective throughput and success probability, Minstrel algorithm updates the retry chain for the next 100ms interval.

\subsection{Enhanced Minstrel Implementation}

In order to increase the efficiency of Minstrel algorithm in the coexistence scenario, we have modified some features of the Minstrel algorithm as our last contribution in this work. First of all, the starting rate for a transmission is chosen based on the SNR at association time using Table 4.2. The purpose is to choose a starting rate 
Table 4.2: Starting Rate to SNR association

\begin{tabular}{c|c} 
MCS & SNR \\
\hline \hline 0 & 2 \\
\hline 1 & 5 \\
\hline 2 & 9 \\
\hline 3 & 11 \\
\hline 4 & 15 \\
\hline 5 & 18 \\
\hline 6 & 20 \\
\hline 7 & 25 \\
\hline 8 & 29 \\
\hline 9 & 31
\end{tabular}

that is reliable so we don't have to rely on sampling to converge to a working rate. All lower rates are given a $100 \%$ starting probability and all higher rates are given a $0 \%$ starting probability.

Secondly, we replace the fourth rate (base rate) with the second highest probability rate. The table 4.3 shows the selection of rates in the Enhanced Minstrel. The reason for this change is that in the coexistence scenario we anticipate to have a lot of collisions. Original Minstrel can not differentiate between the failures due to collisions and failures due to bad channel conditions. Thus, it would falsely reduce the transmission rate in the case of collisions while the channel condition may be good. On the other hand, transmission with the base rate is taking a longer period compared to the higher rates resulting in a bigger probability of failure due to collision. Therefore, employing the second highest probability rate can considerably increase the Minstrel's performance in the coexistence. In our particular implementation we set all count pairs equal to 2 giving a maximum number of retransmissions up to 8 . Finally, as the last improvement, we propose a round robin selection of rates in the sampling mode with initializing at the highest rate to faster build a good statistics 
Table 4.3: Enhanced Minstrel Retry Chain (T: highest throughput, t: second highest throughput, P: highest probability, P-1: Second highest probability, S: sample rate)

\begin{tabular}{c|c|c|c|c} 
Retry Index & 1 & 2 & 3 & 4 \\
\hline \hline No Sampling & $\mathrm{T}$ & $\mathrm{t}$ & $\mathrm{P}$ & $\mathrm{P}-1$ \\
\hline Sample Higher & $\mathrm{S}$ & $\mathrm{T}$ & $\mathrm{t}$ & $\mathrm{P}$ \\
\hline Sample Lower & $\mathrm{T}$ & $\mathrm{S}$ & $\mathrm{t}$ & $\mathrm{P}$ \\
\hline
\end{tabular}

for different available rates.

\subsection{Mutual Information Implementation}

For LTE-LAA the Mutual Information (MI) algorithm is selected as the link adaptation approach. MI algorithm tries to find out the optimal CQI index within four major steps: 1) SINR calculations, 2) SINR to MI mapping, 3) MI to efficiency mapping and 4) Efficiency to CQI.

\section{SINR Calculation}

The very first step for starting the MI algorithm is the SINR computation per subcarrier. For this step we assume that we have knowledge of the noise variance and the channel matrix.

\section{SINR to MI mapping}

The second step in MI algorithm is the SINR to MI mapping, which actually shows how many information bits a symbol can support in an AWGN channel. Such a mapping is provided in Figure 4.1. So after calculating the average SINR per subcarrier, we find out which Modulation scheme actually results the highest MI. After that the selected modulation is used by the BS for the 


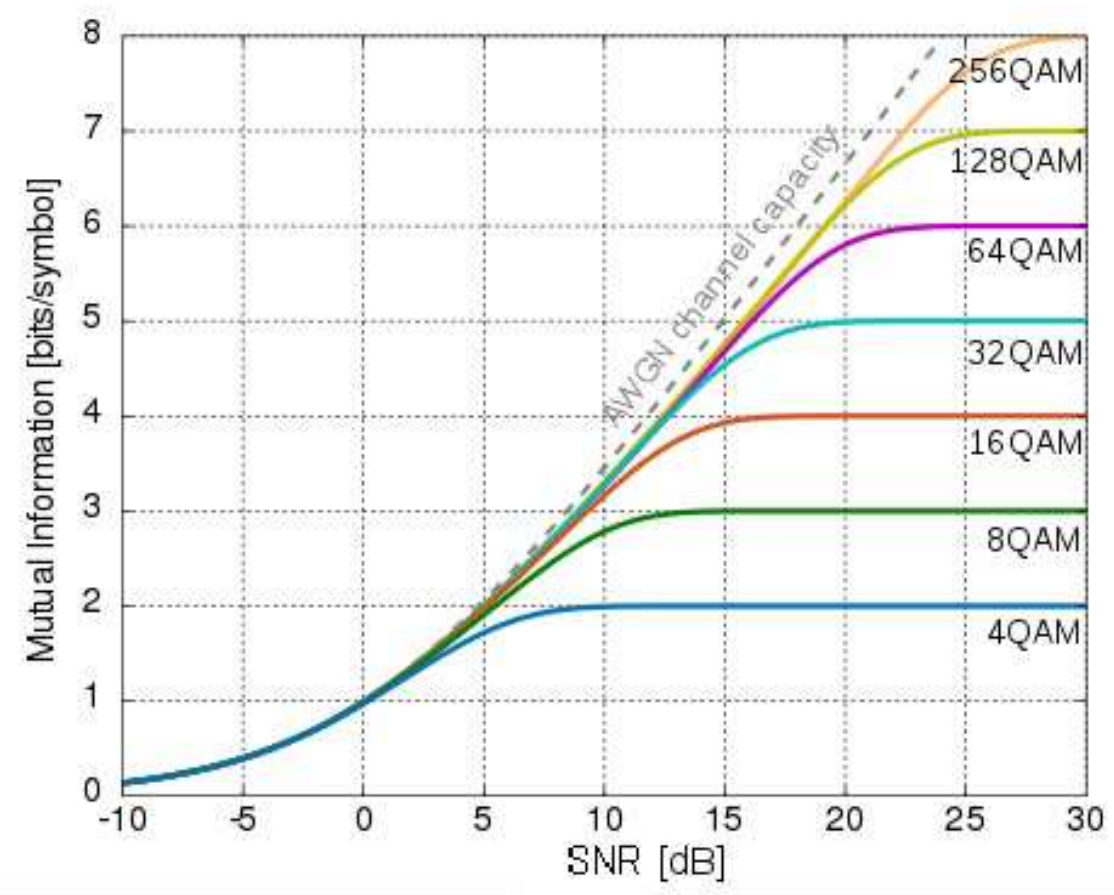

Figure 4.1: SNR to Mutual Information Mapping

new rate.

\section{MI to efficiency mapping}

Figure 4.1 provides an ideal mapping between the SINR and MI. However, because of non-ideal decoding, in practice the actual information bits that the channel can transmit is less. For this reason, a second mapping takes place called MI to efficiency mapping. The gap between the ideal mapping and the second mapping relies on both the code block size and the target BLER needed for transmission.

\section{Efficiency to CQI}

The computed efficiency is used to get a proper transport block size with respect 
to the tables provided by the $3 \mathrm{GPP}$ [40], by considering the number of Resource Blocks (RBs) assigned to the transmission. Following, the obtained Transport Block Size (TBS) efficiency is rounded down to the nearest efficiency in Table 2.1 to choose the optimal CQI.

\subsection{Results and Discussions}

In this section, we evaluate the efficiency of the link adaptations algorithms for different communication conditions. Two experiments are carried out: i) in the first experiment we evaluate the performance of our enhanced Minstrel algorithm over the original Minstrel while, ii) in the second experiment we explore the coexistence of WiFi and LAA when Link Adaptation is activated and when not. The experiments are arranged as follows:

\section{Experiment 1}

An extended number of simulations were carried out to evaluate the performance increase achieved when using the enhanced Minstrel algorithm. Only WiFi is considered in this set of experiments. The enhanced Minstrel algorithm is compared against a basic Minstrel implementation presented in [46] and a WiFi operation with no link adaptation activated using a fixed MCS index of 3. Specifically, we evaluate the achieved throughput and packet error rate performance when changing i) the distance between the AP and the stations, and ii)the number of stations associated with the AP.

\section{Experiment 2}

The second set of experiments consider the coexistence of WiFi and LAA. The goal is to identify possible problems in performance when the two technologies operate together, and what are the benefits of activating the link adaptation in their performance. Similar, we evaluate the performance achieved mainly in terms of throughput when changing i) the distance between the AP/BS and stations/UEs, and ii)the number of stations/UEs associated with the AP/BS. 

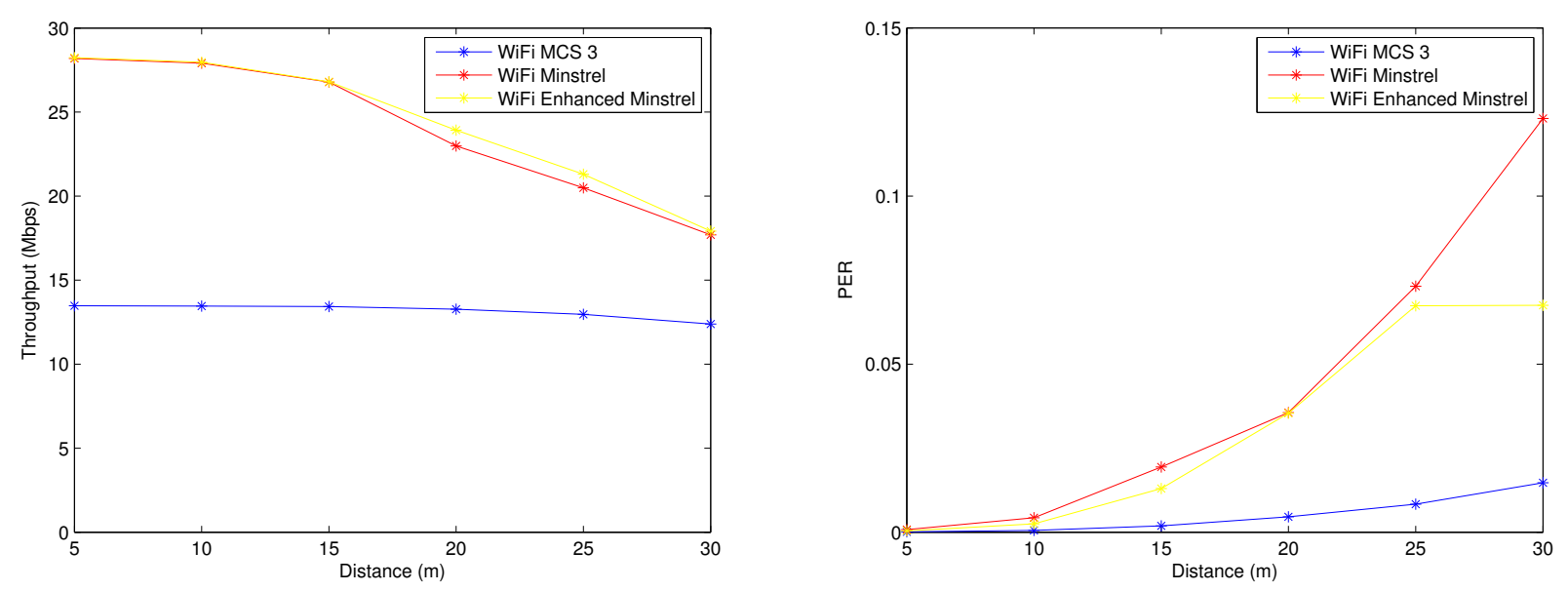

Figure 4.2: Minstrel performance in terms of a) Throughput and b) PER with increasing distance

\subsubsection{Performance Verification of Link Adaptation in WiFi (1 AP 1 STA)}

First we evaluate the performance of the enhanced Minstrel implementation, when increasing the distance between the station and the AP from $5 \mathrm{~m}$ to $30 \mathrm{~m}$ with a step of 5 .

As was expected the throughput drops with the distance and the Packet Error Rate (PER) increases. Our proposed Minstrel implementation, however shows a better performance especially after $15 \mathrm{~m}$ distance. The enhancements proposed make Minstrel more robust when the distance increases by finding a better rate than the common Minstrel implementation. The better performance is illustrated both in terms of throughput and PER in Figure 4.2. Specifically, the enhanced Minstrel depicts an increase in throughput of about $1 \mathrm{Mbps}$ and a decrease in PER of about $1 \%$. On the other hand, when we do not consider any link adaptation scheme (blue line in Figure 8) the throughput remains almost stable for the different examined distances with a small decrease in throughput for $25 \mathrm{~m}$ and $30 \mathrm{~m}$ which is corroborated by the increase noticed in PER. 

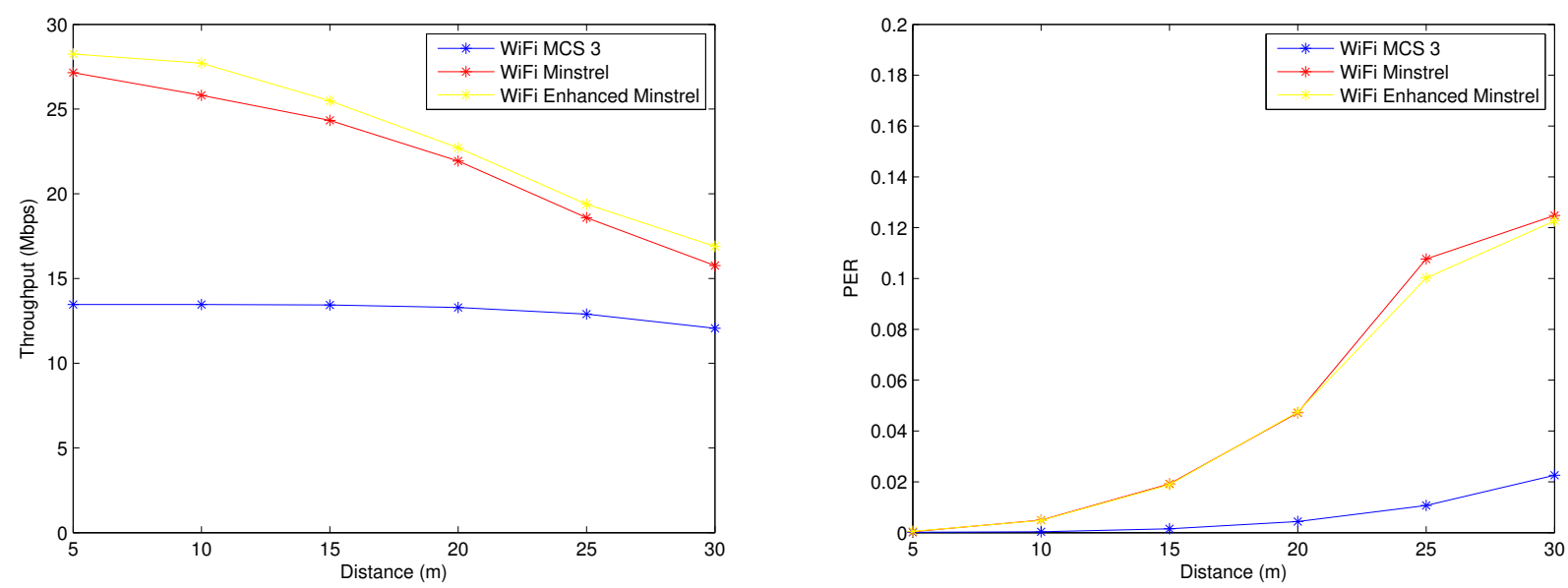

Figure 4.3: Minstrel performance in terms of a) Throughput and b) PER with increasing distance

\subsubsection{Performance Verification of Link Adaptation in WiFi (1 AP 5 stations)}

In this part we repeat the set of experiment presented in the previous Section, however we consider 5 stations associated with the AP instead of 1 . The goal is to validate the performance of the enhanced Minstrel implementation in case we have collisions to see how the algorithm adapts not only in terms of the channel conditions but also in terms of congestion in the network. Figure 4.3 depicts the performance achieved when changing the distance.

Figure 4.3 corroborate the results presented in the previous experiment. In particular, for all the examined set of parameters the enhanced Minstrel implementation shows higher performance by increasing the overall system throughput and decreasing the average PER. Furthermore, we notice that this time for all the examined ranges the increase is much more obvious. This reveals the fact that the enhanced Minstrel increase the WiFi performance both in terms of changing the channel conditions and in case more collisions occur during the transmissions. This behavior is desirable in case WiFi coexists with the LAA LTE. 

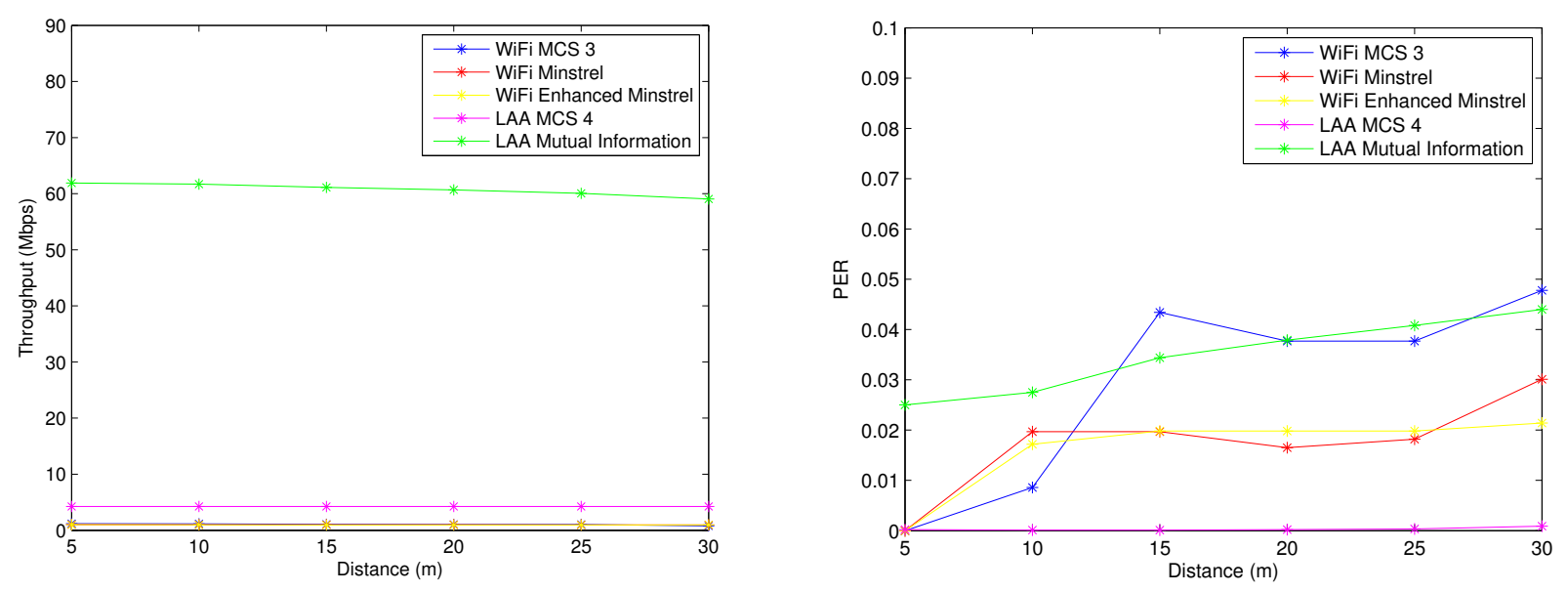

Figure 4.4: WiFi and LAA coexistence performance in terms of a) Throughput and b) PER with increasing distance

\subsubsection{Performance Evaluation of WiFi and LAA coexistence (1 AP/BS 1 station/UE)}

In this section, we also include the LAA LTE during the simulations. In the following, we evaluate the performance achieved for changing the distance again. However, we do not only evaluate the performance of the enhanced Minstrel implementation but also the coexistence aspects for WiFi with LAA LTE.

Figure 4.4 shows the performance achieved when WiFi coexists with LAA LTE and the distance is increased. Six different cases are examined:

a) WiFi without considering any link adaptation scheme using an MCS index of 3, coexisting with LAA LTE using also a fixed MCS index of 4.

b) WiFi operating with Enhanced Minstrel along with LAA LTE using a fixed MCS index of 4.

c) WiFi operating with base Minstrel along with LAA LTE using a fixed MCS index of 4 .

d) WiFi without considering any link adaptation scheme using an MCS index of 3 coexisting with LAA LTE using the Mutual Information algorithm.

e) WiFi operating with Enhanced Minstrel along with LAA LTE using Mutual Information. 
f) WiFi operating with base Minstrel along with LAA LTE using Mutual Information.

For cases a-c the LAA performs exactly the same and that is why there is only one line describing the performance for LAA. Similarly, the same performance is extracted for cases d-f for LAA with Mutual Information. The specific behavior means that the LAA for the current experimentation set up does not seem to be affected by the WiFi regardless of its mode of operation. However, the most noticeable observation in Figure 4.4 is that now the implementation of Minstrel algorithm does not contribute to the overall performance at all. Actually, all three different operation of WiFi present a similar behavior, while in terms of PER the enhanced Minstrel implementation presents the lowest PER. On the other hand, the LAA manages to perform higher throughput performance with very low PER. The reason, behind this behavior is that LTE is a much more efficient technology than WiFi and when LAA gains access to the channel manages to transmit more packets in its transmission time increasing the throughput achieved and reducing PER. Finally, when the Mutual Information is activated in LAA the throughput performance is considerably boosted validating the high efficiency of the link adaptation algorithm. As expected the high throughput achieved by Mutual Information also leads to a higher PER indicating the fact that some packets need to be lost before finding the optimal rate.

However, it is worth noticing that even in these conditions the enhanced Minstrel implementation can provide a marginal increase both in terms of throughput and PER and especially in extreme communication conditions (e.g. 30m distance). Furthermore, as in case with only WiFi the performance of LAA follows the channel and communication conditions. For example, the achieved throughput is decreased with increasing distance.

\subsubsection{Performance Evaluation of WiFi and LAA coexistence (1 AP/BS 5 stations/UEs)}

In this part we repeat the set of experiments presented in the previous sub-section, however we consider 5 stations associated with the AP and 5 UEs associated with the BS. Figures 4.5 depicts the performance achieved when changing the distance.

As with the case of a single communicating device with each AP/BS the same performance is observed when 5 users are associated with the AP and BS. The LAA 

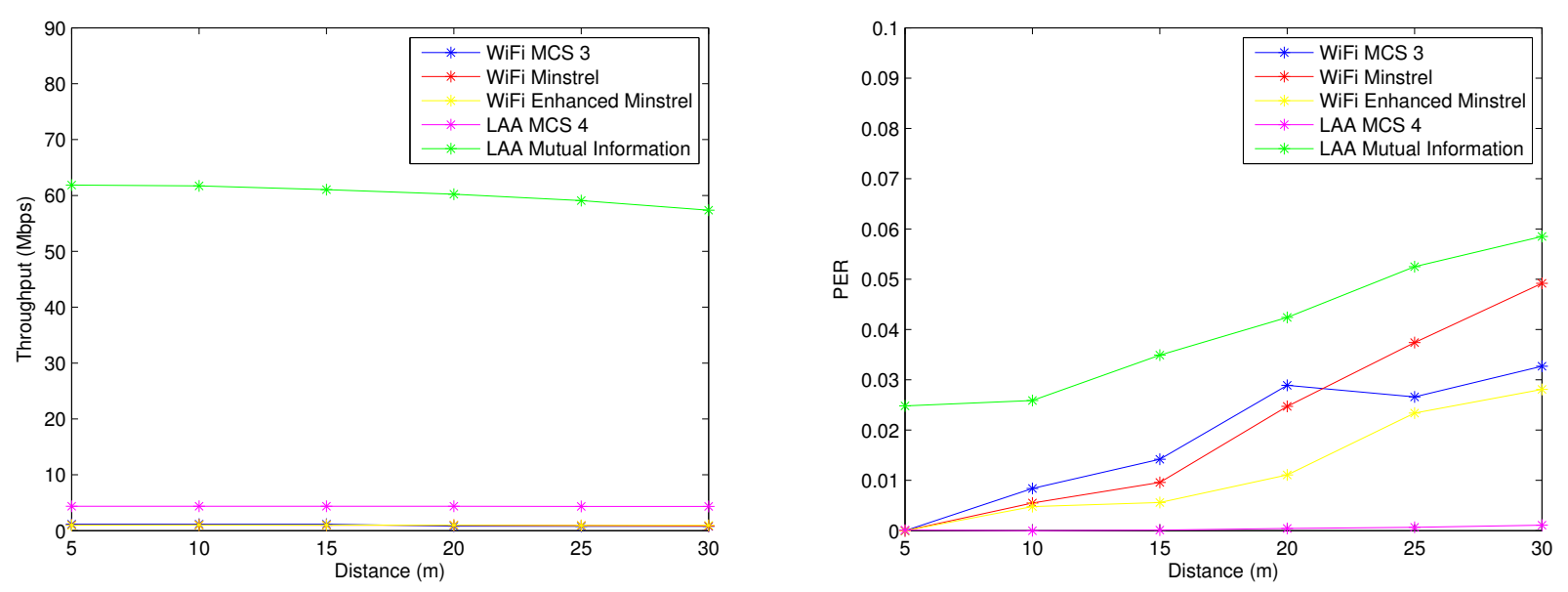

Figure 4.5: WiFi and LAA coexistence performance in terms of a) Throughput and b) PER with increasing distance

with Mutual Information remains to achieve the best system performance (sum of the individual throughput for each UE/STA) for all the examined parameters, while still the LAA operation outperforms the WiFi regardless the operating mode. Again, LAA with Mutual Information manages to succeed a higher throughput performance but presenting at the same time a higher BLER than the other cases.

From the above results it is understood that a number of limitations exist that restrict the performance achieved in WiFi. In particular, we can see that the attained throughput is reduced from around $28 \mathrm{Mbps}$ when we have only WiFi operating with Minstrel algorithm, to $1 \mathrm{Mbps}$ in case of coexistence with LAA. Furthermore, in the latter case Minstrel algorithm is not able to contribute in the overall performance. By getting a closer look in the simulation results it can be understood that the number of transmissions over the 10 seconds period that the simulations last, the number of successful transmissions drops from 40000 to only 1200 when LAA is activated. From this behavior we can easily interpret that LAA presents a dominant channel occupancy leading to severe throughput degradation for WiFi.

Thus, appropriate alterations in the operation of WiFi should be considered towards enabling a harmonic coexistence between WiFi and LAA. To this end, possible improvements that may be considered are:

1. Enable smarter sampling in Minstrel algorithm by allowing to cycle through the available rates faster. 
2. Change the long term probability factor when calculating the EWMA probability of success

3. Sample higher rates more often to move to higher rates faster.

4. Find the optimal distance between AP and BS to maximize throughput and PER performance.

5. Reduce the allocated transmission time for LAA LTE.

6. Increase the packet size used for WiFi.

The 1-3 propositions may yield to better results but they involve a considerable overhead that actually may not be applicable in a real environment. Besides, the main problem identified is the performance degradation due to the increased number of collisions and the excessive amount of channel occupancy that LAA causes. Towards this extend, we examine the 4-6 propositions only.

\subsubsection{Equalizing performance in $\mathrm{WiFi}$ and LAA coexistence}

In the first set of experiments of this section we increase the distance between the $\mathrm{AP}$ and the BS and we evaluate the performance achieved. The results are shown in Figure 4.6, where the offset distance between the AP and BS increases from 10 to $100 \mathrm{~m}$ with a step of $10 \mathrm{~m}$.

From the Figure 4.6 we can draw the following conclusions. For the specific set-up the performance of LAA seems to be unaffected from WiFi for the different distances between AP and BS. Furthermore, the performance of WiFi starts to increase for an offset distance of $90 \mathrm{~m}$ and only at $100 \mathrm{~m}$ the performance achieved is similar with the one attained when only WiFi is operating. In the same manner, PER starts decreasing when the offset distance increases as less collisions are happening in the medium. The small increase observed for the Minstrel algorithm for 90 and $100 \mathrm{~m}$ is due to the fact that now the packet losses are not caused by the collisions but due to the fact the Minstrel algorithm actually can now sample more rates that do not necessary lead to a successful transmission. However, the specific solution is not applicable to a real environment, where the AP and BS are supposed to coexist together in close proximity (i.e. office environment). 

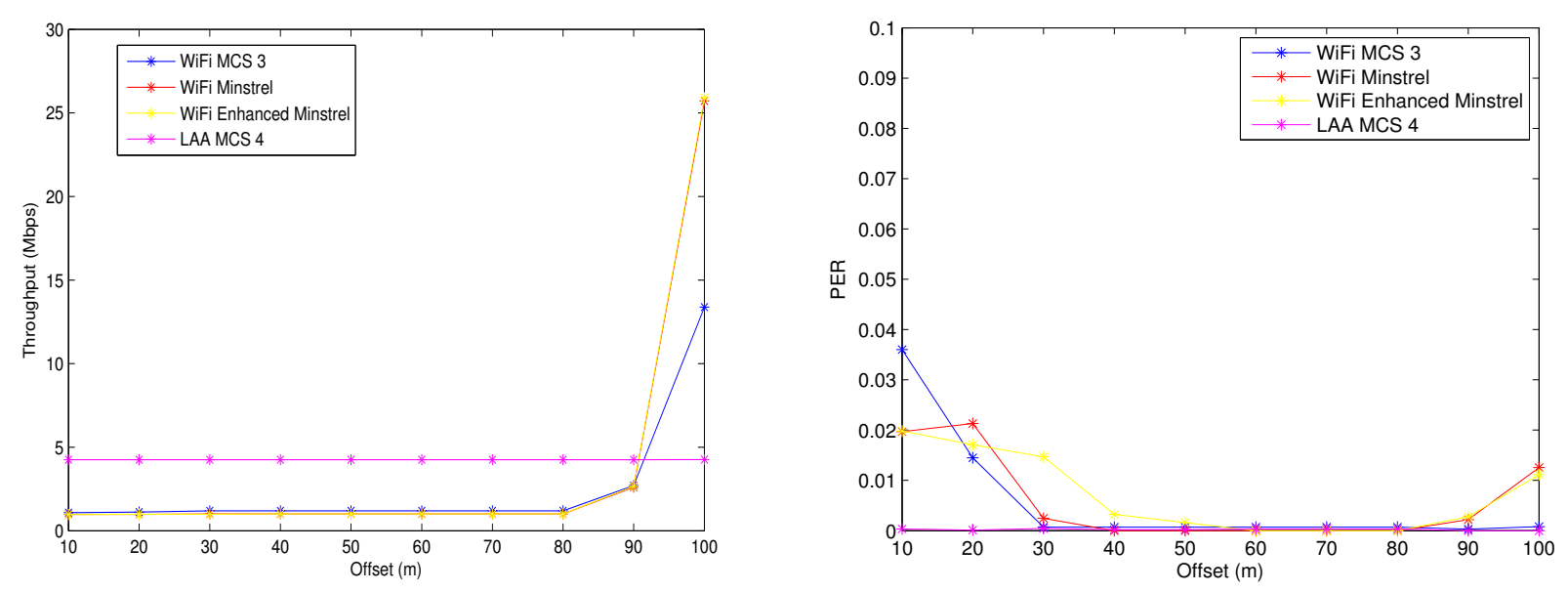

Figure 4.6: WiFi and LAA coexistence performance in terms of a) Throughput and b) PER with increasing offset distance between AP and BS

In the second set of experiments the transmission time of LAA is reduced. In the previous experiments we considered that once LAA gains access to the medium a maximum transmission of $4 \mathrm{~ms}$ occurs or 4 subframes are transmitted. In the following, we examine how the attained throughput changes when LAA transmits for shorter times (i.e. for 2 subframes and 1 subframe or $2 \mathrm{~ms}$ and $1 \mathrm{~ms}$ respectively). For the following results only the distance between the communicating devices with the AP and BS is assumed to change. Finally, to better illustrate the difference in throughput between WiFi and LAA we do not consider the activation of the Mutual Information algorithm for LAA.

From Figures 4.7 and 4.8 we can see that the gap in the performance between WiFi and LAA is decreased as the allocated transmission time for LAA is reduced. Actually, the throughput for WiFi is slightly higher than the throughput for LAA when we consider LAA transmissions with only one subframe. However, even in this case Minstrel is not able to contribute in the overall throughput performance. The reason, is that again the number of transmissions for $\mathrm{WiFi}$, and thus the load, is very low and cannot take advantage of the higher rates that can be selected from Minstrel algorithm. Once again, the enhanced Minstrel implementation can provide a marginal performance improvement compared with the base Minstrel.

As proved in Chapter 3, in order to achieve a fair coexistence between $\mathrm{WiFi}$ and 

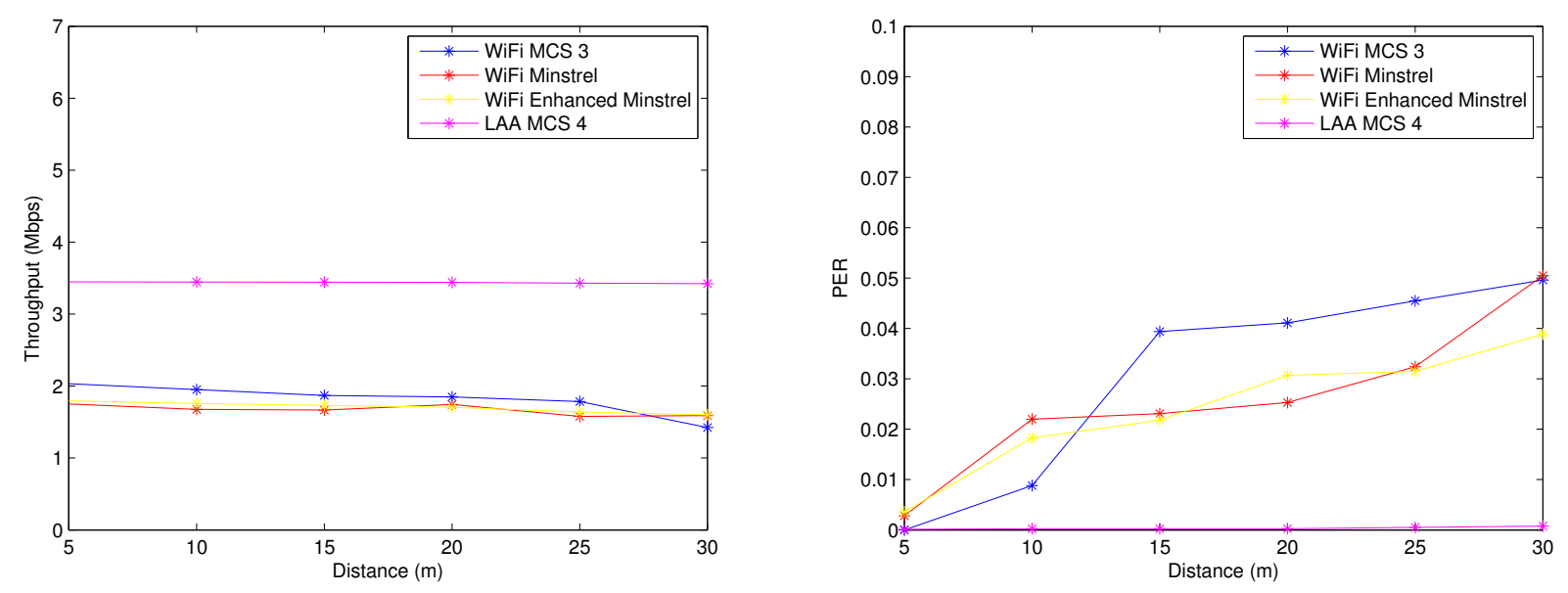

Figure 4.7: WiFi and LAA coexistence performance in terms of a) Throughput and b) PER with LAA sending 2 subframes.
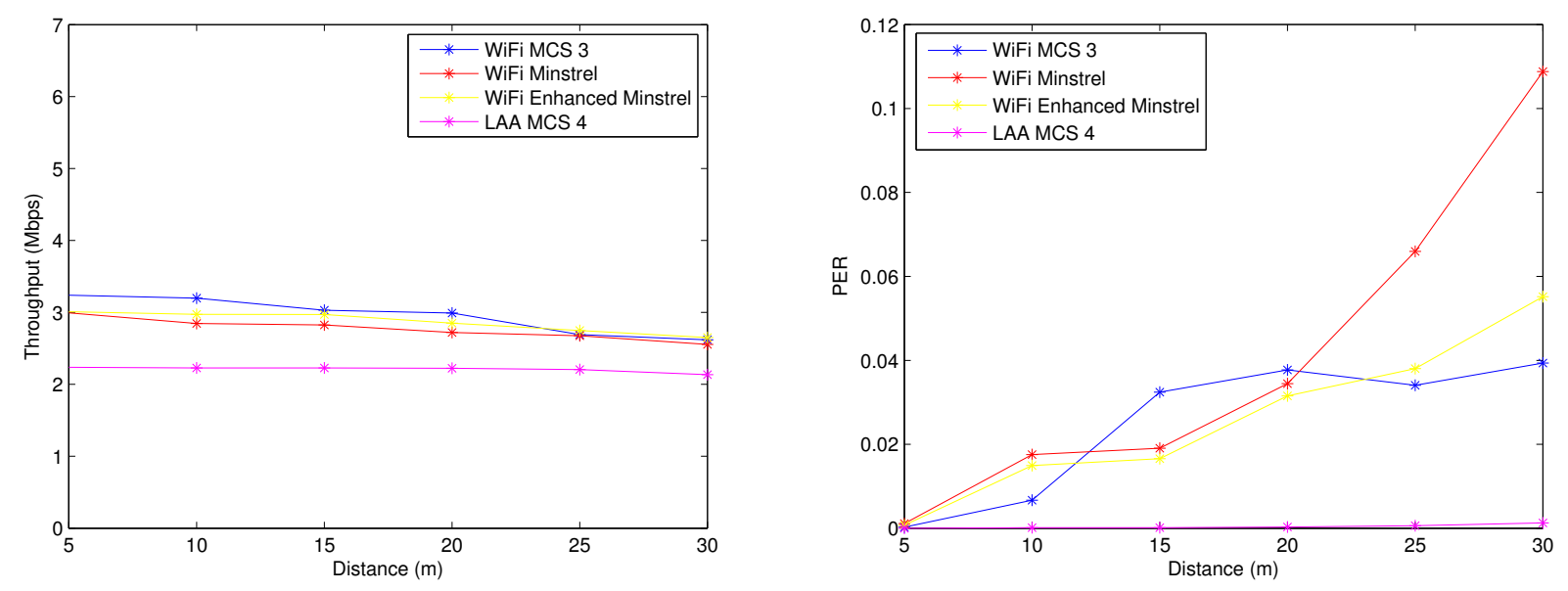

Figure 4.8: WiFi and LAA coexistence performance in terms of a) Throughput and b) PER with LAA sending 1 subframes. 

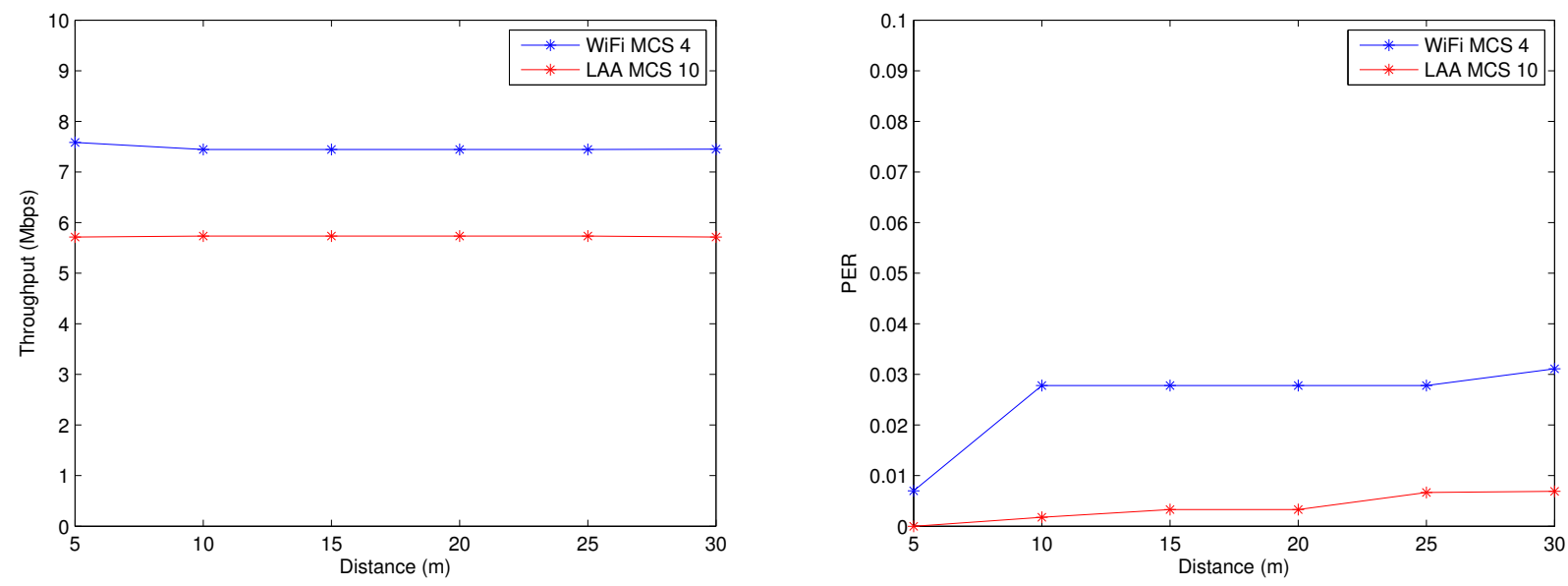

Figure 4.9: WiFi and LAA coexistence performance in terms of a) Throughput and b) PER with no link adaptation activated and with equal transmission times

LAA we need to increase the packet length and so the transmission time. To this end, for the final set of simulations, we increase the transmission time of WiFi to $1 \mathrm{msec}$ and allow LAA to transmit one subframe to equalize the transmission time between the two technologies to make the channel occupancy fair .

Figure 4.9 illustrates the performance of the two technologies when equal transmissions are allowed for the AP and the BS. In this particular simulation we do not consider any link adaptation scheme for either WiFi or LAA. Furthermore, we use an appropriate MCS index for both of them that results in a similar modulation order and coding rate. From the results we see that now, WiFi performs better in terms of throughput achieving a higher rate of around 1.5 Mbps than LAA. However, the higher rate causes an increase in the PER in regards with the LAA. Overall, the throughput capabilities of the medium seems to be equally distributed between the two technologies, without any of the two technologies dominating on the channel. This behavior corroborates the result extracted in chapter 3. In particular, by increasing the packet length of WiFi we can succeed a similar throughput performance with LAA-LTE and guarantee a graceful coexistence.

The last simulation includes the activation of link adaptation for both WiFi and LAA. Again we examine the performance achieved for an increasing distance between the communicating devices with the AP/BS. As we can see from Figure 4.10, the LAA through the Mutual Information algorithm can increase the performance considerably 

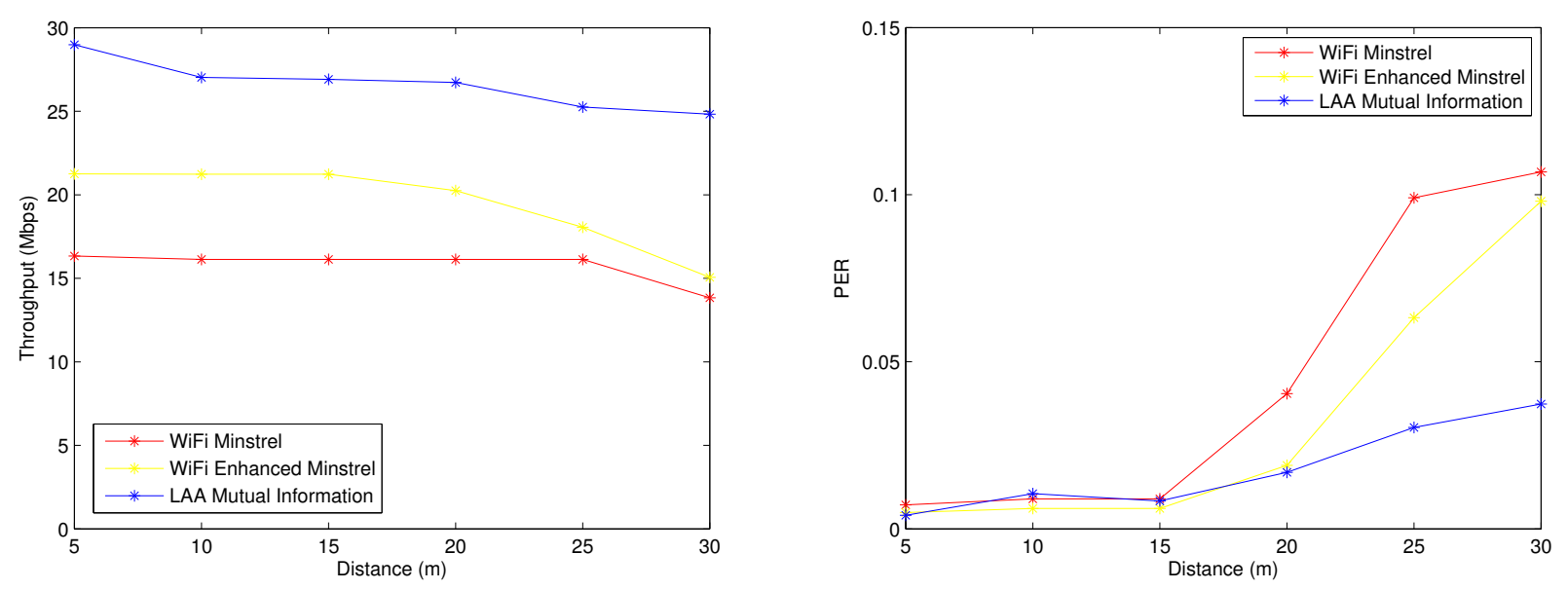

Figure 4.10: WiFi and LAA coexistence performance in terms of a) Throughput and b) PER with link adaptation activated and with equal transmission times

more than WiFi. This has to do with the fact that link adaptation algorithms for LTE are much more efficient than WiFi. Mutual Information algorithm is more dynamic and adaptive than Minstrel, since it manages to change the MCS after every transmission by providing the necessary feedback to the BS. On the other hand, Minstrel updates his retry chain every $0.1 \mathrm{sec}$ which is not that often in a scenario were full buffer is assumed and packets are continuously generated from the AP. Furthermore, link adaptation in LTE offers more granularity since there is a wider range of MCS indexes that may be selected in comparison with only 8 MCS Indexes that Minstrel can select in the particular simulations. However, even in this scenario the Enhanced Minstrel algorithm manages to present a higher throughput performance than the base Minstrel, validating the fact that it performs better in an environment with high probability of collisions. It is worth to mention that around $70 \%$ of the performance improvement in the enhanced Minstrel is due to the second improvement (substitution of base rate with the second highest probability rate in the last trial). The first and last modifications on the other hand, are only responsible for the remaining $30 \%$ of the improvement. These portions of improvements are highly dependent of the length of simulation. For example for a shorter simulation, the effect of the second improvement will reduce, while the remaining two improvements will grow in importance. 


\section{Chapter 5}

\section{Conclusion and Future Works}

As a conclusion, fair coexistence of WiFi-LAA is very essential for both technologies to have a satisfactory share of the unlicensed band. Currently, the proposed schemes for LBT/LAA can outperform $\mathrm{WiFi}$ in utilizing the channel, and therefore cannot guarantee the WiFi fair share from the network. In this work, we have investigated two important notions of fairness in the attained throughput for both networks. The two fairness criteria are the equal throughput for WiFi and LAA separately, and the equal throughput per number of stations communicating in them. Our numerical experiments included changing the maximum packet size both optimally and heuristically for the WiFi network to achieve a fair share from the network in the saturation condition. Moreover, we analyzed the user allocation to both networks (assuming users are equipped with transceivers and receivers from both technologies) in order to achieve a fair coexistence in terms of achievable throughput.

Our results indicate that by proper tuning the maximum packet size for the WiFi network a fair share for the WiFi network is achievable. Also, proper allocation of adaptable users within the two technologies can further increase the fairness for the LAA/WiFi coexistence. This allocation of users can be performed by an information exchange within the $\mathrm{WiFi}$ access point and the base station through the control links at the cost of small signaling overhead.

Finally, we investigated the link adaptation technique to increase the performance of WiFi network. We proposed an enhanced Minstrel algorithm to improve the WiFi's

performance when operating alone and in the case of coexistence. Our findings for the first case, not only indicated that the proposed enhanced Minstrel outperforms the original Minstrel, but also improves the WiFi's performance significantly compared to the case that no link adaptation is activated. However, inspired by the numerical 
analysis we validated the fact that by increasing the packet length of WiFi a considerable increase of WiFi throughput can be achieved. Specifically, when the packet length of the two technologies are equalized the enhanced Minstrel implementation can increase the overall throughput by 5 Mbps.

In the performed experiments, we have changed the distance between the communicating devices both in WiFi and LTE-LAA. We tried to illustrate how the performance changes when WiFi AP and the LTE eNB are further apart from their associated devices. We also considered the effects for different distances of WiFi $\mathrm{AP}$ and LTE eNB. However, the distance is not the only indicator of the network performance. There exists several other important disregarded factors such as the transmission power, speed of the users, different channel environment (e.g. office versus an open area) and etc. that play an important role in this coexistence. Moreover we only considered the small networks in our analysis with small number of stations due to the simplicity of our model. Therefore, the behavior of larger networks stayed uninvestigated. Hopefully, we will cover these remaining aspects in our future work. 


\section{List of References}

[1] G. Bianchi, "Performance analysis of the ieee 802.11 distributed coordination function," IEEE Journal on selected areas in communications, vol. 3, pp. 535$547,2000$.

[2] Y. Gao, X. Chu, and J. Zhang, "Performance analysis of laa and wifi coexistence in unlicensed spectrum based on markov chain," in Global Communications Conference (GLOBECOM), pp. 1-6, 2016.

[3] H. Cengis, M. K. Marina, and U. Challita, "On lte-wifi coexistence and interoperator spectrum sharing in unlicensed bands: altruism, cooperation and fairness," in In Proceedings of the 17th ACM International Symposium on Mobile Ad Hoc Networking and Computing, pp. 111-120, 2016.

[4] A. Babaei, J. Andreoli-Fang, Y. Pang, and B. Hamzeh, "On the impact of lte-u on wi-fi performance," International Journal of Wireless Information Networks, vol. 22, pp. 336-344, 2015.

[5] "Lte in unlicensed spectrum: Harmonious coexistence with wi-fi." https://www . qualcomm.com/documents/lte-unlicensed-coexistence-whitepaper. Accessed: 2017-02-20.

[6] "Lte-u technology and coexistence." http://www.Iteuforum.org/uploads/ 3/5/6/8/3568127/lte-u_coexistence_mechansim_qualcomm_may_28_2015. pdf. Accessed: 2017-02-21.

[7] W. Xuyu, S. Mao, and M. X. Gong, "A survey of lte wi-fi coexistence in unlicensed bands," GetMobile: Mobile Computing and Communications, vol. 20, pp. 17-23, 2017.

[8] A. Mukherjee, J. Cheng, S. Falahati, H. Koorapaty, R. Karaki, L. Falconetti, and D. Larsson, "Licensed-assisted access lte: Coexistence with ieee 802.11 and the evolution toward 5g," IEEE Communications Magazine, vol. 54, pp. 50-57, 2016.

[9] H. J. Zhu and D. Kidston, "The impact of link adaptation on wifi 802.11 n," in Networking, Architecture and Storage (NAS), pp. 1-5, 2016.

[10] S. Wu, S. Biaz, B. Qi, and K. Zhang, "Bara: a sender based rate adaptation in wireless networks," in Proceedings of the 45th annual southeast regional conference, pp. 179-184, 2007. 
[11] R. Gummadi, D. Wetherall, B. Greenstein, and S. Seshan, "Understanding and mitigating the impact of rf interference on 802.11 networks," ACM SIGCOMM Computer Communication, vol. 37, pp. 385-396, 2007.

[12] X. Zhang and K. G. Shin, "Enabling coexistence of heterogeneous wireless systems: Case for zigbee and wifi," in Proceedings of the Twelfth ACM International Symposium on Mobile Ad Hoc Networking and Computing, p. 6, 2011.

[13] J. Zhu, A. Waltho, X. Yang, and X. Guo, "Multi-radio coexistence: Challenges and opportunities," in Computer Communications and Networks, 200\%. ICCCN 200\%. Proceedings of 16th International Conference on IEEE, pp. 358-364, 2007.

[14] M. A. Cavalcante, E. Almedia, R. D. Vieira, F. Chavez, R. Paiva, F. Abinader, S. Choudhuri, E. Tuomaala, and K. Doppler, "Performance evaluation of lte and wi-fi coexistence in unlicensed bands," in Vehicular Technology Conference (VTC Spring) IEEE rrth, pp. 1-6, 2013.

[15] T. Nihtila, V. Tykhomyrov, O. Alanen, M. A. Uusitalo, A. Mangalvedhe, M. Moisio, S. Iraji, R. Ratasuk, and N. Mangalvedhe, "System performance of lte and ieee 802.11 coexisting on a shared frequency band," in Wireless Communications and Networking Conference (WCNC), pp. 1038-1043, 2013.

[16] R. Ratasuk, M. A. Uusitalo, N. Mangalvedhe, A. Sorri, S. Iraji, C. Wijting, and A. Ghosh, "License-exempt lte deployment in heterogeneous network," in International Symposium on Wireless Communication Systems (ISWCS), pp. 246$250,2012$.

[17] R. C. Paiva, P. Papadimitriou, and S. Choudhury, "A physical layer framework for interference analysis of lte and wi-fi operating in the same band," in Signals, Systems and Computers, on Asilomar Conference, pp. 1204-1209, 2013.

[18] S. F. Chaves, E. Almedia, R. D. Vieira, A. M. Cavalcante, F. M. Abinader, S. Choudhury, and K. Doppler, "Lte ul power control for the improvement of lte/wi-fi coexistence," in Vehicular Technology Conference (VTC Fall), IEEE 78th, pp. 1-6, 2013.

[19] S. Sagari, S. Baysting, D. Saha, I. Seskar, W. Trappe, and D. Raychaudhuri, "Coordinated dynamic spectrum management of lte-u and wi-fi networks," in Dynamic Spectrum Access Networks (DySPAN), IEEE International Symposium on, pp. 209-220, 2015.

[20] E. Almeida, A. M. Cavalcante, R. C. Paiva, S. Chaves, F. M. Abinader, R. D. Vieira, S. Choudhury, E. Tuomaala, and K. Doppler, "Enabling lte/wifi coexistence by lte blank subframe allocation," in Communications (ICC) IEEE International Conference on, pp. 5083-5088, 2013.

[21] H. Zhang, X. Chu, W. Guo, and S. Wang, "Coexistence of wi-fi and heterogeneous small cell networks sharing unlicensed spectrum," IEEE Communications Magazine, vol. 53, pp. 158-164, 2015. 
[22] S. Wang, W. Guo, and M. D. McDonnell, "Downlink interference estimation without feedback for heterogeneous network interference avoidance," in Telecommunications (ICT), 21st International Conference on, pp. 82-87, 2014.

[23] C. Cano and D. Leith, "Unlicensed lte/wifi coexistence: Is lbt inherently fairer than csat?," in Communications (ICC), IEEE International Conference, pp. 1-6, 2016.

[24] C. Cano and D. Leith, "Coexistence of wifi and lte in unlicensed bands: A proportional fair allocation scheme," in IEEE ICC 2015 Workshop on LTE in Unlicensed Bands: Potentials and Challenges, London, UK, pp. 2288-2293, 2015.

[25] X. Wang, Q. S. Tony, M. Sheng, and J. Li, "Throughput and fairness analysis of wi-fi and lte-u in unlicensed band.," in IEEE Journal on Selected Areas in Communications 35.1, pp. 63-78, 2017.

[26] G. Holland, N. Vaidya, and P. Bahl, "A rate-adaptive mac protocol for multi-hop wireless networks," in Proceedings of the 7th annual international conference on Mobile computing and networking. ACM, pp. 236-251, 2001.

[27] B. Sadeghi, V. Kanodia, and A. Sabharwal, "Opportunistic media access for multirate ad hoc networks," in Proceedings of the 8th annual international conference on Mobile computing and networking. ACM, pp. 24-35, 2002.

[28] S. H. Wong, H. Yang, S. Lu, and V. Bharghavan, "Robust rate adaptation for 802.11 wireless networks," in Proceedings of the 12th annual international conference on Mobile computing and networking. ACM, pp. 146-157, 2006.

[29] A. Kamerman and L. Monteban, "Wavelan ii: A high-performance wireless lan for the unlicensed band," Bell Labs Technical Journal, vol. 2, pp. 118-133, 1997.

[30] M. Lacage, M. Manshaei, and T. Turletti, "Ieee 802.11 rate adaptation: A practical approach," in Proceedings of the 7th ACM international symposium on Modeling, analysis and simulation of wireless and mobile systems, p. 126 134, 2004.

[31] J. Kim, S. Kim, S. Choi, and D. Qiao, "Cara: Collision-aware rate adaptation for ieee 802.11 wlans," Infocom, vol. 6, pp. 1-11, 2006.

[32] "Madwifi." http://sourceforge.net/projects/madwifi. Accessed: 2017-0226.

[33] J. Bicket, "Bit-rate selection in wireless networks," in MIT Master's Thesis, 2005.

[34] "Minstrel specification, madwifi project."

[35] F. Tramarin, S. Vitturi, and M. Luvisotto, "Improved rate adaptation strategies for real-time industrial ieee $802.11 \mathrm{n}$ wlans," in IEEE 20th conference on Emerging Technologies and Factory Automation(ETFA), pp. 1-8, 2015.

[36] X. Chen, H. Yi, H. Luo, and H. Yu, "A novel cqi calculation scheme in lte and ltea systems," in Proceedings of the IEEE International Wireless Communications and Signal Processing (WCSP), pp. 1-5, 2011. 
[37] J. Jian, M. Peng, Y. Li, and Y. Wei, "Measurement-based optimizing algorithm for modulation and coding scheme selection in downlink lte self-organizing networks," in Proceedings of the IEEE International Conference on Wireless Information Technology and Systems (ICWITS), pp. 1-4, 2012.

[38] M. Shahab, "Efficient channel quality indicator reporting schemes in lte with reduced signalling overhead," in the 38th International Conference on Telecommunications and Signal Processing (TSP), pp. 123-128, 2015.

[39] S. N. Donthi and N. Mehta, "An accurate model for eesm and its application to analysis of cqi feedback schemes and scheduling in lte," IEEE Transactions on Wireless Communications, vol. 10, pp. 3436-3448, 2011.

[40] "Lte: Evolved universal terrestrial access (e-utra); physical layer procedures." ETSI TS 136213 V12.4.0.

[41] K. Sayana and J. Zhuang, "Link performance abstraction based on mean mutual information per bit (mmib) of the llr channel," in IEEE 802.16 Broadband Wireless Access Working Group, Standardization Document, 2007.

[42] 3rd Generation Partnership Project TR 36.889, "Study on licensed-assisted access to unlicensed spectrum; (release 13)," 2015.

[43] B. Chen, J. Chen, Y. Gao, and J. Zhang, "Coexistence of lte-laa and wi-fi on 5 ghz with corresponding deployment scenarios: A survey," IEEE Communications Surveys and Tutorials 19.1, vol. 1, pp. 7-32, 2017.

[44] C. Cano, D. Lopez-Perez, H. Claussen, and D. J. Leith, "Using lte in unlicensed bands: potential benefits and coexistence issues," in IEEE Communications Magazine 54.12, pp. 116-123, 2016.

[45] D. Xia, J. Hart, and Q. Fu, "On the performance of rate control algorithm minstrel," in Personal Indoor and Mobile Radio Communications (PIMRC), pp. 406$412,2012$.

[46] D. Xia, J. Hart, and Q. Fu, "Evaluation of the minstrel rate adaptation algorithm in ieee 802.11g wlans," in Proceedings of the IEEE International Conference on Communications (ICC), pp. 2223-2228, 2013. 